\title{
Spectral enclosures for a class of block operator matrices
}

\author{
Juan Giribet ${ }^{\mathrm{a}, \mathrm{b}}$, Matthias Langer ${ }^{\mathrm{c}}$, Francisco Martínez Pería ${ }^{\mathrm{a}, \mathrm{d}, *}$, Friedrich Philipp ${ }^{\mathrm{e}}$, Carsten Trunk ${ }^{\mathrm{a}, \mathrm{f}}$ \\ aInstituto Argentino de Matemática “Alberto P. Calderón” (CONICET), Saavedra 15 (1083) Buenos Aires, Argentina \\ ${ }^{b}$ Departamento de Matemática - FI-Universidad de Buenos Aires, Paseo Colón 850 (1063) Buenos Aires, Argentina \\ ${ }^{c}$ Department of Mathematics and Statistics, University of Strathclyde, 26 Richmond Street, Glasgow G1 1XH, United Kingdom \\ personal. strath. ac. uk/m. langer \\ ${ }^{d}$ Centro de Matemática de La Plata - FCE, Universidad Nacional de La Plata, C.C. 172, (1900) La Plata, Argentina \\ ${ }^{e}$ Katholische Universität Eichstätt-Ingolstadt, Ostenstraße 26, 85072 Eichstätt, Germany \\ www. ku. de/?fmphilipp \\ Institut für Mathematik, Technische Universität Ilmenau, Postfach 100565, D-98684 Ilmenau, Germany \\ www. tu-ilmenau. de/de/analysis/team/carsten-trunk
}

\begin{abstract}
We prove new spectral enclosures for the non-real spectrum of a class of $2 \times 2$ block operator matrices with self-adjoint operators $A$ and $D$ on the diagonal and operators $B$ and $-B^{*}$ as off-diagonal entries. One of our main results resembles Gershgorin's circle theorem. The enclosures are applied to $J$-frame operators.
\end{abstract}

Keywords: Block operator matrices, quadratic numerical range, spectral enclosure, Gershgorin's circle

\section{Introduction}

We consider block operator matrices $S$ acting in the orthogonal sum $\mathscr{H}:=\mathscr{H}_{+} \oplus \mathscr{H}_{-}$of two Hilbert spaces,

$$
S=\left[\begin{array}{cc}
A & B \\
-B^{*} & D
\end{array}\right]
$$

where $A$ and $D$ are (possibly unbounded) self-adjoint operators in $\mathscr{H}_{+}$and $\mathscr{H}_{-}$, respectively, and $B$ is a bounded operator from $\mathscr{H}_{-}$to $\mathscr{H}_{+}$.

Such operators play an important role in various applications. For instance, they appear in the study of so-called floating singularities [6, 14, 15, 19, 21], in the perturbation theory for equations of indefinite Sturm-Liouville type [5], and also in frame theory [12, 13].

Clearly, $S$ is not self-adjoint in $\mathscr{H}$ unless $B=0$. However, it is self-adjoint if we introduce the indefinite inner product

$$
\left[\left[\begin{array}{c}
x_{+} \\
x_{-}
\end{array}\right],\left[\begin{array}{l}
y_{+} \\
y_{-}
\end{array}\right]\right]=\left(x_{+}, y_{+}\right)-\left(x_{-}, y_{-}\right), \quad\left[\begin{array}{l}
x_{+} \\
x_{-}
\end{array}\right],\left[\begin{array}{l}
y_{+} \\
y_{-}
\end{array}\right] \in \mathscr{H}
$$

for bounded $S$ this means that $[S x, y]=[x, S y]$ for all $x, y \in \mathscr{H}$. The indefinite inner product $[\cdot, \cdot]$ turns $\mathscr{H}$ into a Krein space, i.e. it is the orthogonal sum of a Hilbert space and an anti-Hilbert space. Actually, every bounded self-adjoint operator in a Krein space can be written in the form 1.1.

\footnotetext{
*Corresponding author

Email addresses: jgiribet@fi.uba.ar (Juan Giribet), m.langer@strath.ac.uk (Matthias Langer), francisco@mate.unlp.edu.ar (Francisco Martínez Pería ), fmphilipp@gmail.com (Friedrich Philipp), carsten.trunk@tu-ilmenau.de (Carsten Trunk)
} 
Of particular interest is the location of the spectrum of $S$. In [18, 23, 30] spectral enclosures were obtained via the quadratic numerical range, and in [4, 5] in terms of the spectra of $A$ and $D$. Gershgorintype results for more general operator matrices were presented in [9] and [28]. Moreover, in [1, 14, 20, 21] the essential spectrum was investigated, and in [19] variational principles and estimates for eigenvalues were proved. Invariant subspaces and factorizations of Schur complements were considered in [24] and [2], and in [3] conditions were presented for an operator of the form (1.1) to be similar to a self-adjoint operator in a Hilbert space. For an overview we refer to the monograph [29].

In general, the spectrum of block operator matrices as in (1.1) is not contained in the real line. The self-adjointness of $S$ in the Krein space with the inner product 1.2 implies only that the spectrum of $S$ is symmetric with respect to the real axis. The aim of this paper is to prove enclosures for the (non-real) spectrum of $S$ in terms of (spectral) quantities of the operators $A, B$, and $D$.

We start with a general enclosure for the (closure) of the quadratic numerical range of $S$, formulated in terms of the numerical ranges of $A$ and $D$ and the norm of $B$; see Proposition 3.1 below. The quadratic numerical range of a block operator matrix was introduced in [22] and its closure contains the spectrum of $S$; see 2.2. Although similar enclosures for the spectrum of $S$ were already known, one of the advantages of having a spectral enclosure for the quadratic numerical range is that it leads also to estimates of the norm of the resolvent; see the discussion in Remark 3.2 Moreover, Proposition 3.1 is sharp in the sense that the enclosures for the quadratic numerical range cannot be improved if just the numerical ranges of $A$ and $D$ and the norm of $B$ are known; see Theorem 3.3 .

The main contribution of this paper is a spectral enclosure for the operator matrix $S$, which is connected with the Schur complements. It is well known and follows from a relatively simple Neumann series type argument applied to the first and second Schur complement that

$$
\sigma(S) \backslash \mathbb{R} \subseteq\left\{\lambda \in \mathbb{C} \backslash \mathbb{R}:\left\|B^{*}(A-\lambda)^{-1} B(D-\lambda)^{-1}\right\| \geq 1 \text { and }\left\|B(D-\lambda)^{-1} B^{*}(A-\lambda)^{-1}\right\| \geq 1\right\} ;
$$

see [9, Theorem 1.1], [4, Lemma 5.2(ii)] or [29, Section 2.3]. Here we prove that

$$
\sigma(S) \backslash \mathbb{R} \subseteq\left\{\lambda \in \mathbb{C} \backslash \mathbb{R}:\left\|(A-\lambda)^{-1} B\right\| \geq 1 \quad \text { and } \quad\left\|(D-\lambda)^{-1} B^{*}\right\| \geq 1\right\} ;
$$

see Theorem 4.3 below. The enclosures 1.3 and 1.4 are independent of each other in the sense that none of the sets in the right-hand sides of $(1.3)$ and $(1.4)$ is strictly contained in the other one. However, since the norm inequalities in (1.4) deal separately with the resolvent functions of $A$ and $D$ it is easy to construct examples where the enclosure in (1.4) is strictly contained in the one in $(1.3)$, see Example 4.9 .

Note that the spectral enclosures in $(1.3)$ and $(1.4)$ are not explicitly formulated in terms of the spectra of $A$ and $D$. However, it is one of our main observations that (1.4) allows a reformulation in a more geometric manner. In particular, given $\lambda \in \mathbb{C} \backslash \mathbb{R},\left\|(A-\lambda)^{-1} B\right\| \geq 1$ if and only if for all positive continuous functions $f: \mathbb{R} \rightarrow \mathbb{R}$ with some specific behaviour at infinity we have

$$
\lambda \in \bigcup_{t \in \sigma_{B}(A)} \mathscr{B}_{f(t)^{-1}\|f(A) B\|}(t),
$$

where $\sigma_{B}(A)$ is a specific closed subset of $\sigma(A)$ and $\mathscr{B}_{r}(t)$ stands for the closed ball of radius $r$ around $t$; for details we refer to Proposition 4.7 below. A similar interpretation can be obtained for the inequality $\left\|(D-\lambda)^{-1} B^{*}\right\| \geq 1$ in terms of continuous functions defined in a closed subset $\sigma_{B^{*}}(D)$ of the spectrum of $D$. Therefore, the enclosure for the non-real part of the spectrum of $S$ in (1.4) implies a family of enclosures which resemble Gershgorin's circle theorem: for any two positive continuous functions $f$ and $g$ with some specific behaviour at infinity we have that

$$
\sigma(S) \backslash \mathbb{R} \subseteq\left(\bigcup_{t \in \sigma_{B}(A)} \mathscr{B}_{f(t)^{-1}\|f(A) B\|}(t)\right) \cap\left(\bigcup_{s \in \sigma_{B^{*}}(D)} \mathscr{B}_{g(s)^{-1}\left\|g(D) B^{*}\right\|}(s)\right) ;
$$


see Theorem 4.8 below.

Maybe the most interesting situations appear when one chooses the functions $f$ and $g$ explicitly. For instance, if $A$ is boundedly invertible and $f(t)=|t|^{-1}$ then (1.5) implies

$$
\sigma(S) \backslash \mathbb{R} \subseteq \bigcup_{a \in \sigma_{B}(A)} \mathscr{B}_{|a|\left\|A^{-1} B\right\|}(a) .
$$

Moreover, if $\left\|A^{-1} B\right\|<1$ then these balls are contained in a double-sector with half opening angle $\arcsin \left\|A^{-1} B\right\|$, see Figure 1 below.

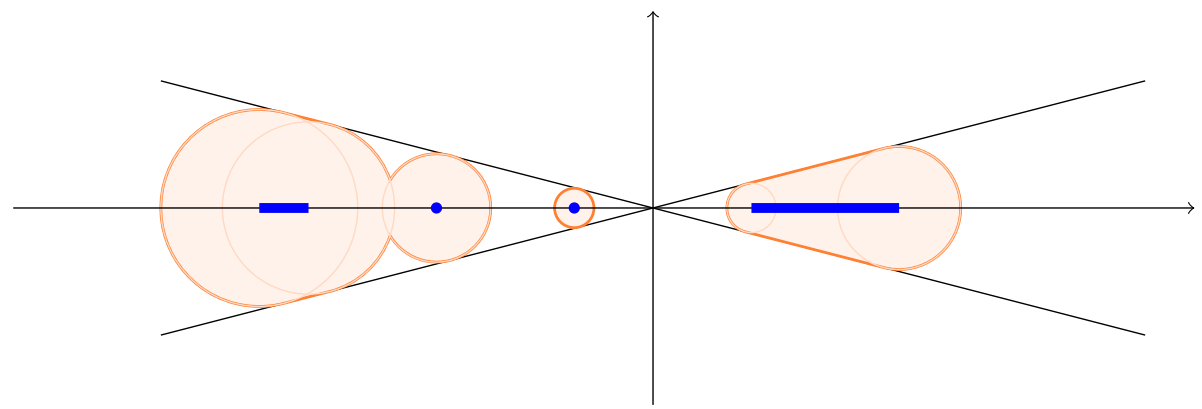

Figure 1: The spectral enclosure for $\sigma(S) \backslash \mathbb{R}$ given in 1.6 (in orange) and the set $\sigma_{B}(A)$ (in blue).

It is worth mentioning that (1.4) also improves the following spectral enclosure obtained in [5]: if $\mathscr{B}_{r}(\Delta)=\{z \in \mathbb{C}: \operatorname{dist}(z, \Delta) \leq r\}$ then

$$
\sigma(S) \backslash \mathbb{R} \subseteq \mathscr{B}_{\|B\|}(\sigma(A)) \cap \mathscr{B}_{\|B\|}(\sigma(D)) .
$$

In fact, $\left\|(A-\lambda)^{-1} B\right\| \geq 1$ obviously implies $\left\|(A-\lambda)^{-1}\right\|\|B\| \geq 1$ and the latter is equivalent to $\lambda \in$ $\mathscr{B}_{\|B\|}(\sigma(A))$. A similar argument with $D$ and $B^{*}$ instead of $A$ and $B$ completes the proof.

Finally, in Section 5 we apply the spectral enclosures obtained in Section 4 to operator matrices of the form (1.1) which appear in frame theory. The so-called $J$-frame operators were introduced in [13] and further investigated in [12]. Our findings lead to significant improvements of the spectral enclosures for $J$-frame operators obtained in [12].

\section{Preliminaries}

If $\mathscr{H}$ and $\mathscr{K}$ are Hilbert spaces, we denote by $L(\mathscr{H}, \mathscr{K})$ the space of all bounded linear operators mapping from $\mathscr{H}$ to $\mathscr{K}$. As usual, we set $L(\mathscr{H}):=L(\mathscr{H}, \mathscr{H})$. For $r \geq 0$ and $\Delta \subseteq \mathbb{C}$ we set

$$
\mathscr{B}_{r}(\Delta):=\{z \in \mathbb{C}: \operatorname{dist}(z, \Delta) \leq r\} .
$$

If $a \in \mathbb{C}$, we also write $\mathscr{B}_{r}(a):=\mathscr{B}_{r}(\{a\})$ for the closed disc with centre $a$ and radius $r$.

The numerical range of a linear operator $T$ in the Hilbert space $\mathscr{H}$ is defined by

$$
W(T):=\{(T x, x): x \in \operatorname{dom} T,\|x\|=1\} .
$$

It is well known that the numerical range $W(T)$ is convex and that $\mathbb{C} \backslash \overline{W(T)}$ has at most two (open) connected components (see [16, V.3.2]). Moreover, it is immediate from the definition of $W(T)$ that $\sigma_{\mathrm{p}}(T) \subseteq W(T)$, where $\sigma_{\mathrm{p}}(T)$ stands for the point spectrum of $T$. If $T$ is closed, $\lambda \in \mathbb{C} \backslash \overline{W(T)}$ and 
$x \in \operatorname{dom} T,\|x\|=1$, then

$$
\|(T-\lambda) x\| \geq|(T x, x)-\lambda| \geq \operatorname{dist}(\lambda, \overline{W(T)}) .
$$

This shows that $\operatorname{ran}(T-\lambda)$ is closed and $\operatorname{ker}(T-\lambda)=\{0\}$. Hence, if each of the (at most two) components of $\mathbb{C} \backslash \overline{W(T)}$ contains points from the resolvent set $\rho(T)$, then $\sigma(T) \subseteq \overline{W(T)}$. This holds in particular if $T$ is bounded. The next lemma is now immediate.

Lemma 2.1. Let $T=A+B$, where $A$ is a self-adjoint operator in a Hilbert space $\mathscr{H}$ and $B \in L(\mathscr{H})$. Then $\sigma(T) \subseteq \overline{W(T)}$ and for $\lambda \notin \overline{W(T)}$ we have

$$
\left\|(T-\lambda)^{-1}\right\| \leq \frac{1}{\operatorname{dist}(\lambda, \overline{W(T)})} .
$$

Let us also recall the definition of the quadratic numerical range, which was introduced in [22]; see also [29. Definition 1.1.1]. Assume that the Hilbert space $\mathscr{H}$ is the orthogonal sum of two Hilbert spaces, $\mathscr{H}_{+}$and $\mathscr{H}_{-}$. Let $S$ be a bounded operator in $\mathscr{H}$ decomposed as

$$
S=\left[\begin{array}{cc}
A & B \\
C & D
\end{array}\right]
$$

where $A \in L\left(\mathscr{H}_{+}\right), B \in L\left(\mathscr{H}_{-}, \mathscr{H}_{+}\right), C \in L\left(\mathscr{H}_{+}, \mathscr{H}_{-}\right)$, and $D \in L\left(\mathscr{H}_{-}\right)$. For $f \in \mathscr{H}_{+}$and $g \in \mathscr{H}_{-}$with $\|f\|=\|g\|=1$ we introduce the $2 \times 2$ matrix

$$
S_{f, g}=\left[\begin{array}{ll}
(A f, f) & (B g, f) \\
(C f, g) & (D g, g)
\end{array}\right] .
$$

The set

$$
W^{2}(S):=\bigcup_{\substack{f \in \mathscr{H}_{+}, g \in \mathscr{H}_{-} \\\|f\|=\|g\|=1}} \sigma_{\mathrm{p}}\left(S_{f, g}\right)
$$

is called the quadratic numerical range of $S$. It is no longer a convex subset of $\mathbb{C}$, but it has at most two connected components.

One of the advantages of the quadratic numerical range is that it is contained in the numerical range: $W^{2}(S) \subseteq W(S)$ and that we have the following refined spectral inclusions

$$
\sigma_{\mathrm{p}}(S) \subseteq W^{2}(S) \quad \text { and } \quad \sigma(S) \subseteq \overline{W^{2}(S)} ;
$$

see [29. Theorem 1.3.1]. Moreover, the resolvent can be estimated in terms of the distance to $W^{2}(S)$ :

$$
\left\|(S-\lambda)^{-1}\right\| \leq \frac{\|S\|+|\lambda|}{\left[\operatorname{dist}\left(\lambda, W^{2}(S)\right)\right]^{2}}, \quad \lambda \notin \overline{W^{2}(S)} ;
$$

see [29. Theorem 1.4.1]. If $\overline{W^{2}(S)}=F_{1} \cup F_{2}$ with disjoint non-empty closed sets $F_{1}$ and $F_{2}$, then

$$
\left\|(S-\lambda)^{-1}\right\| \leq \frac{\|S\|+|\lambda|}{\operatorname{dist}\left(\lambda, F_{1}\right) \operatorname{dist}\left(\lambda, F_{2}\right)}, \quad \lambda \notin \overline{W^{2}(S)} ;
$$

see [29, Theorem 1.4.5].

The quadratic numerical range definition can be easily extended to unbounded block operator matrices, restricting the vectors $f$ and $g$ in 2.1 to the proper domains $(\operatorname{dom} A) \cap(\operatorname{dom} C)$ and $(\operatorname{dom} B) \cap(\operatorname{dom} D)$, 
respectively. For details see [29, Definition 2.5.1].

In the following we recall the definition of the Schur complements of a block operator matrix, which are powerful tools to study the spectrum and spectral properties. Let $A$ and $D$ be closed operators in $\mathscr{H}_{+}$ and $\mathscr{H}_{-}$, respectively, $B \in L\left(\mathscr{H}_{-}, \mathscr{H}_{+}\right)$, and $C \in L\left(\mathscr{H}_{+}, \mathscr{H}_{-}\right)$. For the block operator matrix

$$
S=\left[\begin{array}{ll}
A & B \\
C & D
\end{array}\right], \quad \operatorname{dom} S=\operatorname{dom} A \oplus \operatorname{dom} D,
$$

the first and second Schur complements of $S$ are defined by:

$$
\begin{array}{ll}
S_{1}(\lambda):=A-\lambda-B(D-\lambda)^{-1} C, & \lambda \in \rho(D), \\
S_{2}(\lambda):=D-\lambda-C(A-\lambda)^{-1} B, & \lambda \in \rho(A) .
\end{array}
$$

These are analytic operator functions defined on the resolvent sets of $D$ and $A$, respectively.

In the next sections we shall make use of the following auxiliary result from [25]; see also [29. Theorem 2.3.3].

Lemma 2.2 ([25, Theorem 2.4]). Let $A$ and $D$ be closed operators in $\mathscr{H}_{+}$and $\mathscr{H}_{-}$, respectively, let $B \in L\left(\mathscr{H}_{-}, \mathscr{H}_{+}\right)$and $C \in L\left(\mathscr{H}_{+}, \mathscr{H}_{-}\right)$, and consider the block operator matrix

$$
S=\left[\begin{array}{ll}
A & B \\
C & D
\end{array}\right], \quad \operatorname{dom} S=\operatorname{dom} A \oplus \operatorname{dom} D .
$$

Then the following statements hold:

(i) For $\lambda \in \rho(D)$ one has $\lambda \in \sigma(S)$ if and only if $0 \in \sigma\left(S_{1}(\lambda)\right)$.

(ii) For $\lambda \in \rho(A)$ one has $\lambda \in \sigma(S)$ if and only if $0 \in \sigma\left(S_{2}(\lambda)\right)$.

Moreover, if $\lambda \in \rho(D) \cap \rho(S)$, then

$$
(S-\lambda)^{-1}=\left[\begin{array}{cc}
I & 0 \\
-(D-\lambda)^{-1} C & I
\end{array}\right]\left[\begin{array}{cc}
S_{1}(\lambda)^{-1} & 0 \\
0 & (D-\lambda)^{-1}
\end{array}\right]\left[\begin{array}{cc}
I & -B(D-\lambda)^{-1} \\
0 & I
\end{array}\right] .
$$

\section{Sharp enclosures for the quadratic numerical range}

Let $S$ be as in 1.1 with bounded operators

$$
A \in L\left(\mathscr{H}_{+}\right), \quad B \in L\left(\mathscr{H}_{-}, \mathscr{H}_{+}\right), \quad D \in L\left(\mathscr{H}_{-}\right),
$$

where $A$ and $D$ are self-adjoint in the Hilbert spaces $\mathscr{H}_{+}$and $\mathscr{H}_{-}$, respectively. Hence, the numerical ranges $W(A)$ and $W(D)$ are real intervals. We introduce the following numbers, which are used for the description of the enclosures that are proved below:

$$
\begin{array}{rlrl}
a_{-}: & : \inf W(A), \\
d_{-}: & :=\inf W(D), & a_{+} & :=\sup W(A), \\
d_{+} & :=\sup W(D), \\
m_{-}:=\frac{a_{-}+d_{-}}{2}, & m_{+}:=\frac{a_{+}+d_{+}}{2}, \\
c:=\frac{1}{2}\left[\min \left\{a_{+}, d_{+}\right\}+\max \left\{a_{-}, d_{-}\right\}\right] & \text {and } \quad \ell:=\frac{1}{2} \operatorname{dist}(\overline{W(A)}, \overline{W(D)})
\end{array}
$$


If $\overline{W(A)} \cap \overline{W(D)}=\varnothing$, then $c$ is the midpoint of the gap between $\overline{W(A)}=\left[a_{-}, a_{+}\right]$and $\overline{W(D)}=\left[d_{-}, d_{+}\right]$, and $\ell$ is half the length of the gap; e.g. if $d_{+}<a_{-}$, then

$$
c=\frac{1}{2}\left(d_{+}+a_{-}\right) \quad \text { and } \quad \ell=\frac{1}{2}\left(a_{-}-d_{+}\right) .
$$

The following proposition contains enclosures of the closure of the quadratic numerical range of operators of the form (1.1). Due to 2.2) these yield also enclosures for the spectrum. Note that the enclosures in Proposition 3.1 depend only on $W(A), W(D)$ and $\|B\|$. They are illustrated in Figures 2,5 below.

Proposition 3.1. Given a Hilbert space $\mathscr{H}=\mathscr{H}_{+} \oplus \mathscr{H}_{-}$, consider the block operator matrix

$$
S:=\left[\begin{array}{cc}
A & B \\
-B^{*} & D
\end{array}\right]
$$

where $B \in L\left(\mathscr{H}_{-}, \mathscr{H}_{+}\right)$, and $A$ and $D$ are bounded self-adjoint operators in $\mathscr{H}_{+}$and $\mathscr{H}_{-}$, respectively. Further, let the constants $a_{ \pm}, d_{ \pm}, m_{ \pm}, c$ and $\ell$ be as in 3.1 3.4 . Then

$$
\begin{aligned}
& \overline{W^{2}(S)} \cap \mathbb{R} \subseteq\left[\min \left\{a_{-}, d_{-}\right\}, \max \left\{a_{+}, d_{+}\right\}\right], \\
& \overline{W^{2}(S)} \backslash \mathbb{R} \subseteq \mathscr{B}_{\|B\|}\left(\left[a_{-}, a_{+}\right]\right) \cap \mathscr{B}_{\|B\|}\left(\left[d_{-}, d_{+}\right]\right) \cap\left\{z \in \mathbb{C}: m_{-} \leq \operatorname{Re} z \leq m_{+}\right\} .
\end{aligned}
$$

Moreover, in the special case where

$$
\overline{W(A)} \cap \overline{W(D)}=\varnothing \quad \text { and } \quad\|B\| \leq \ell,
$$

we have

$$
\overline{W^{2}(S)} \subseteq\left[\min \left\{a_{-}, d_{-}\right\}, c-\sqrt{\ell^{2}-\|B\|^{2}}\right] \cup\left[c+\sqrt{\ell^{2}-\|B\|^{2}}, \max \left\{a_{+}, d_{+}\right\}\right]
$$

in particular, both the quadratic numerical range and the spectrum of $S$ are real.

Proof. Inclusion (3.6) follows from [29, Proposition 1.2.6]. It is sufficient to show (3.7) and (3.9) without the closures on the left-hand sides because the right-hand sides are closed sets. Let $z \in W^{2}(S)$. Then there exist $f \in \mathscr{H}_{+}$and $g \in \mathscr{H}_{-}$with $\|f\|=\|g\|=1$ such that $z$ is an eigenvalue of the matrix $S_{f, g}=\left(\begin{array}{cc}\alpha & \beta \\ -\bar{\beta} & \delta\end{array}\right)$ in (2.1) with $\alpha=(A f, f) \in\left[a_{-}, a_{+}\right], \delta=(D g, g) \in\left[d_{-}, d_{+}\right]$and $\beta=(B g, f)$, which satisfies $|\beta| \leq\|B\|$. By [18, Theorem 2.1] and [5, Theorem 3.5] the inclusion (3.7) holds for $\overline{W^{2}(S)}$ replaced by $\sigma(S)$. Applying this to the matrix $S_{f, g}$ we obtain that in the case when $z$ is non-real, $z$ is contained in

$$
\mathscr{B}_{|\beta|}(\{\alpha\}) \cap \mathscr{B}_{|\beta|}(\{\delta\}) \cap\left\{z \in \mathbb{C}: m_{-} \leq \operatorname{Re} z \leq m_{+}\right\}
$$

and hence in the right-hand side of (3.7).

It remains to show 3.9. We assume without loss of generality that $d_{+}<a_{-}$, in which case $c=$ $\frac{1}{2}\left(a_{-}+d_{+}\right)$and $\ell=\frac{1}{2}\left(a_{-}-d_{+}\right)$. By (3.7), $z \in \mathbb{R}$, and hence $z=z_{+}$or $z=z_{-}$where

$$
z_{ \pm}:=\frac{\alpha+\delta}{2} \pm \sqrt{\left(\frac{\alpha-\delta}{2}\right)^{2}-|\beta|^{2}}
$$

It is easy to see that $z_{+}$is increasing in $\alpha$ and decreasing in $\delta$. Therefore

$$
z_{+} \geq \frac{a_{-}+d_{+}}{2}+\sqrt{\left(\frac{a_{-}-d_{+}}{2}\right)^{2}-|\beta|^{2}} \geq c+\sqrt{\ell^{2}-\|B\|^{2}}
$$


and, similarly, $z_{-} \leq c-\sqrt{\ell^{2}-\|B\|^{2}}$. Together with $(\underline{3.6}$, this shows the inclusion 3.9 .

Remark 3.2. (a) Parts of Proposition 3.1 are known: inclusion [3.6 is from [29, Proposition 1.2.6].

(b) Inclusion (3.7) is an improvement of a similar result in [29, Proposition 1.2.6]. To be more precise, in [29, Proposition 1.2.6] the following inclusion is proved, when $\|B\|>\ell$,

$$
\overline{W^{2}(S)} \backslash \mathbb{R} \subseteq\left\{z \in \mathbb{C}:|\operatorname{Im} z| \leq \sqrt{\|B\|^{2}-\ell^{2}}\right\} \cap\left\{z \in \mathbb{C}: m_{-} \leq \operatorname{Re} z \leq m_{+}\right\} ;
$$

cf. also [29, Proposition 1.3.9]. It is easy to see that the right-hand side of (3.7) is contained in the right-hand side of (3.10), and in most cases the inclusion is strict; see also Figures $2-56$ below.

(c) Inclusion 23.9] improves [29, Proposition 1.2.6] significantly, namely, the former implies that the interval

$$
\left(c-\sqrt{\ell^{2}-\|B\|^{2}}, c+\sqrt{\ell^{2}-\|B\|^{2}}\right)
$$

has empty intersection with $\overline{W^{2}(S)}$ if $\|B\|<\ell$. A similar result for the spectrum of $S$ (which is, in general, a smaller set) can be found in [8, Theorem 4.2] and, in a somehow different form, in [3, Theorem 5.8] and [4, Theorem 5.4].

(d) For enclosures for the quadratic numerical range and the spectrum where all entries $A, B$, and $D$ are allowed to be unbounded see [23, Proposition 4.10 and Theorem 4.13].

Figures 2.5 below show the enclosures for $\overline{W^{2}(S)}$ from Proposition 3.1 In Figures 2 and 3 the situation where $\overline{W(A) \cap W(D)} \neq \varnothing$ is considered. If $\|B\|$ is less than or equal to

$$
\tau:=\min \left\{m_{+}-\min \left\{a_{+}, d_{+}\right\}, \max \left\{a_{-}, d_{-}\right\}-m_{-}\right\},
$$

the non-real spectrum is contained in $\mathscr{B}_{\|B\|}\left(\left[a_{-}, a_{+}\right]\right) \cap \mathscr{B}_{\|B\|}\left(\left[d_{-}, d_{+}\right]\right)=\mathscr{B}_{\|B\|}(\overline{W(A)} \cap \overline{W(D)})$. When $\|B\|>\tau$ then the enclosure in $\left\{z \in \mathbb{C}: m_{-} \leq \operatorname{Re} z \leq m_{+}\right\}$, the third set on the right-hand side of [3.7], has to be taken into account as well.

The case when there is a gap between $\overline{W(A)}$ and $\overline{W(D)}$ is considered in Figures 4 and 5 . When $\|B\|$ is small, then $\overline{W^{2}(S)}$ is contained in the union of the two real intervals on the right-hand side of 3.9 . When $\|B\|$ is larger, then the spectrum may be non-real, and the right-hand sides of (3.6) and (3.7) have to be used.

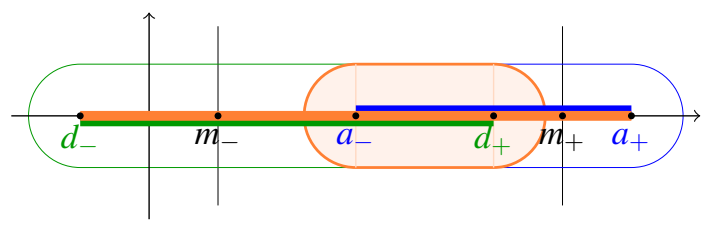

Figure 2: The region (indicated in orange) given by the union of the sets on the right-hand sides of 3.6 and 3.7 that contains $\overline{W^{2}(S)}$, when $\overline{W(A)}$ and $\overline{W(D)}$ overlap and $\|B\|<\tau$. 


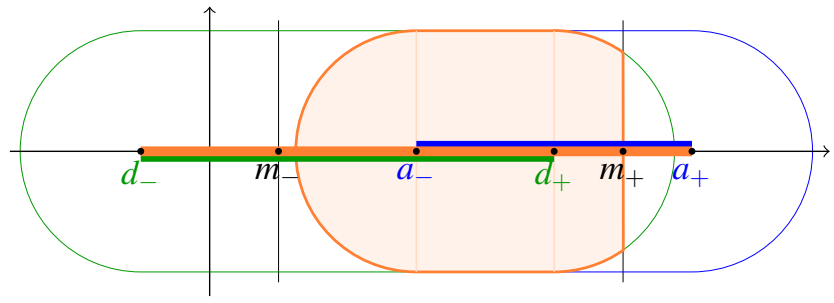

Figure 3: The region (indicated in orange) given by the union of the sets on the right-hand sides of 3.6 and 3.7 that contains $\overline{W^{2}(S)}$, when $\overline{W(A)}$ and $\overline{W(D)}$ overlap and $\|B\|>\tau$.

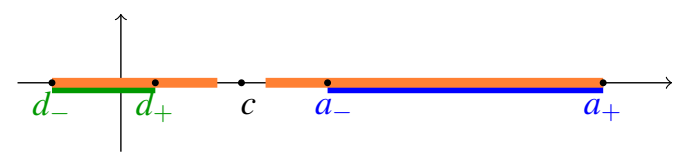

Figure 4: The two intervals (indicated in orange) on the right-hand side of $\overline{3.9}$ whose union contains $\overline{W^{2}(S)}$, when $\overline{W(A)}$ and $\overline{W(D)}$ are separated and $\|B\|<\tau$.

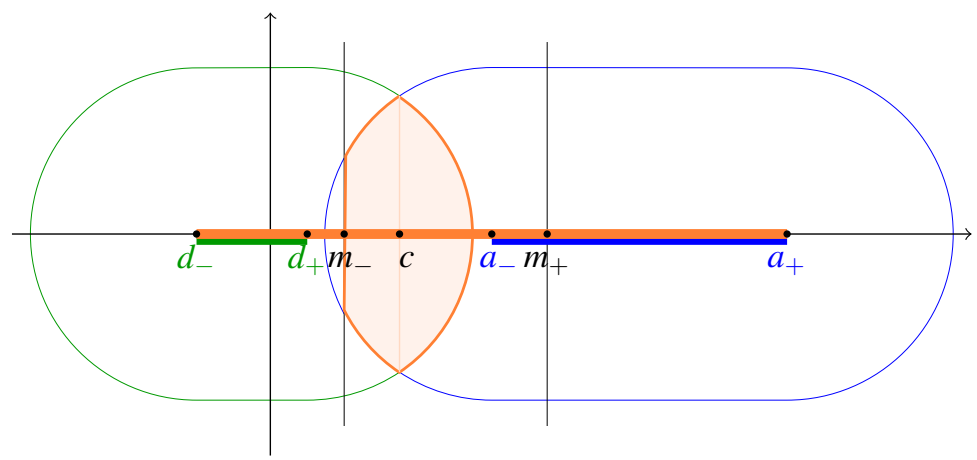

Figure 5: The region (indicated in orange) given by the union of the sets on the right-hand sides of 3.6 and 3.7 that contains $\overline{W^{2}(S)}$, when $\overline{W(A)}$ and $\overline{W(D)}$ are separated and $\|B\|>\tau$.

The next theorem shows that Proposition 3.1 is sharp in the sense that given $\left[a_{-}, a_{+}\right]=\overline{W(A)},\left[d_{-}, d_{+}\right]=$ $\overline{W(D)}$ and $\|B\|$, the enclosures for the spectrum and the quadratic numerical range of $S$ cannot be improved, i.e. an operator $S$ is constructed for which equality holds in 3.6) and (3.7), and in 3.9) if 3.8) is satisfied.

Theorem 3.3. Let $a_{+}, a_{-}, d_{+}, d_{-}, b \in \mathbb{R}$ such that $a_{-} \leq a_{+}, d_{-} \leq d_{+}$, and $b>0$. Then there exist separable Hilbert spaces $\mathscr{H}_{ \pm}$, self-adjoint operators $A$ and $D$ in $\mathscr{H}_{+}$and $\mathscr{H}_{-}$, respectively, and $B \in L\left(\mathscr{H}_{-}, \mathscr{H}_{+}\right)$ such that

$$
\overline{W(A)}=\left[a_{-}, a_{+}\right], \quad \overline{W(D)}=\left[d_{-}, d_{+}\right], \quad\|B\|=b,
$$

and (with the notation from (3.3)-(3.4) the operator $S$ from 3.5 satisfies

$$
\begin{gathered}
\sigma(S)=\overline{W^{2}(S)}=\left(\mathscr{B}_{b}\left(\left[a_{-}, a_{+}\right]\right) \cap \mathscr{B}_{b}\left(\left[d_{-}, d_{+}\right]\right) \cap\left\{z \in \mathbb{C}: m_{-} \leq \operatorname{Re} z \leq m_{+}\right\}\right) \\
\cup\left[\min \left\{a_{-}, d_{-}\right\}, \max \left\{a_{+}, d_{+}\right\}\right]
\end{gathered}
$$


if $b>\ell$ and

$$
\sigma(S)=\overline{W^{2}(S)}=\left[\min \left\{a_{-}, d_{-}\right\}, c-\sqrt{\frac{\ell^{2}}{4}-b^{2}}\right] \cup\left[c+\sqrt{\frac{\ell^{2}}{4}-b^{2}}, \max \left\{a_{+}, d_{+}\right\}\right]
$$

if $b \leq \ell$.

Proof. Let $\mathscr{H}_{+}=\mathscr{H}_{-}=\ell^{2}$ and define the operators

$$
A=\operatorname{diag}\left(a_{1}, a_{2}, \ldots\right), \quad B=\operatorname{diag}\left(b_{1}, b_{2}, \ldots\right), \quad D=\operatorname{diag}\left(d_{1}, d_{2}, \ldots\right)
$$

with numbers

$$
a_{n} \in\left[a_{-}, a_{+}\right], \quad b_{n} \in[0, b], \quad d_{n} \in\left[d_{-}, d_{+}\right],
$$

$n \in \mathbb{N}$, which are chosen later. Let $z_{n}=x_{n}+i y_{n}, n \in \mathbb{N}$, be such that $\left\{z_{n}: n \in \mathbb{N}\right\}$ is a dense subset of the right-hand sides of (3.11) or (3.12), respectively. Below we construct $a_{n}, b_{n}, d_{n}$ such that

$$
z_{n}=w_{+}\left(a_{n}, b_{n}, d_{n}\right) \quad \text { or } \quad z_{n}=w_{-}\left(a_{n}, b_{n}, d_{n}\right),
$$

where

$$
w_{ \pm}\left(a_{n}, b_{n}, d_{n}\right):=\frac{a_{n}+d_{n}}{2} \pm \sqrt{\left(\frac{a_{n}-d_{n}}{2}\right)^{2}-\left|b_{n}\right|^{2}} .
$$

Since then $z_{n}$ is an eigenvalue of $S$ and $\sigma(S)$ is closed, this, together with the enclosures in Proposition 3.1. shows equality in 3.11) and 3.12).

Let us first consider the case when $z_{n} \notin \mathbb{R}$. Then $z_{n}$ is in the right-hand side of [3.11]. If $x_{n} \in\left[a_{-}, a_{+}\right] \cap$ $\left[d_{-}, d_{+}\right]$, then set

Clearly,

$$
a_{n}:=d_{n}:=x_{n}, \quad b_{n}:=\left|y_{n}\right| .
$$

$$
a_{n} \in\left[a_{-}, a_{+}\right], \quad d_{n} \in\left[d_{-}, d_{+}\right], \quad b_{n}=\left|y_{n}\right|=\operatorname{dist}\left(z_{n},\left[a_{-}, a_{+}\right]\right) \leq b,
$$

and (3.14) is satisfied. Now assume that $x_{n} \notin\left[a_{-}, a_{+}\right] \cap\left[d_{-}, d_{+}\right]$. Without loss of generality we can assume that

$$
\operatorname{dist}\left(x_{n},\left[a_{-}, a_{+}\right]\right) \leq \operatorname{dist}\left(x_{n},\left[d_{-}, d_{+}\right]\right),
$$

which implies that $x_{n} \notin\left[d_{-}, d_{+}\right]$. Let us consider the case when $x_{n}<d_{-}$; the case $x_{n}>d_{+}$is analogous. Set

$$
a_{n}=2 x_{n}-d_{-}, \quad d_{n}=d_{-}, \quad b_{n}=\sqrt{y_{n}^{2}+\left(x_{n}-d_{-}\right)^{2}} .
$$

Clearly, $d_{n} \in\left[d_{-}, d_{+}\right]$. From $x_{n} \geq m_{-}$we obtain that

$$
a_{n}=2 x_{n}-d_{-} \geq 2 m_{-}-d_{-}=2 \frac{a_{-}+d_{-}}{2}-d_{-}=a_{-} .
$$

If $x_{n} \leq a_{+}$, then $a_{n}=x_{n}+\left(x_{n}-d_{-}\right)<x_{n} \leq a_{+}$and hence $a_{n} \in\left[a_{-}, a_{+}\right]$. If $x_{n}>a_{+}$, then the inequality in (3.16) is equivalent to $x_{n}-a_{+} \leq d_{-}-x_{n}$, which implies that

$$
a_{n}=2 x_{n}-d_{-} \leq a_{+} ;
$$

hence also in this case we have $a_{n} \in\left[a_{-}, a_{+}\right]$. Moreover,

$$
b_{n}=\sqrt{y_{n}^{2}+\left(x_{n}-d_{-}\right)^{2}}=\left|z_{n}-d_{-}\right|=\operatorname{dist}\left(z_{n},\left[d_{-}, d_{+}\right]\right) \leq b .
$$


It is easy to check that $(3.14)$ is satisfied.

Next we consider the case when $z_{n} \in \mathbb{R}$. If $z_{n} \in\left[a_{-}, a_{+}\right]$, then choose $a_{n}=z_{n}, d_{n}$ arbitrary in $\left[d_{-}, d_{+}\right]$ and $b_{n}=0$. Then

$$
w_{+}\left(a_{n}, b_{n}, d_{n}\right)=\max \left\{z_{n}, d_{n}\right\}, \quad w_{-}\left(a_{n}, b_{n}, d_{n}\right)=\min \left\{z_{n}, d_{n}\right\},
$$

and hence 3.14 holds. The case when $z_{n} \in\left[d_{-}, d_{+}\right]$is similar. If $\left[a_{-}, a_{+}\right] \cap\left[d_{-}, d_{+}\right] \neq \varnothing$, then all cases of real $z_{n}$ are covered. Finally, assume that $\left[a_{-}, a_{+}\right] \cap\left[d_{-}, d_{+}\right]=\varnothing$ and $z_{n} \notin\left[a_{-}, a_{+}\right] \cup\left[d_{-}, d_{+}\right]$. Without loss of generality we can assume that $d_{+}<a_{-}$; then $z_{n} \in\left(d_{+}, a_{-}\right)$. Let us consider the case when $z_{n} \geq c=\frac{1}{2}\left(d_{+}+a_{-}\right)$; the other case is similar. Set

$$
a_{n}=a_{-}, \quad d_{n}=d_{+}, \quad b_{n}=\sqrt{\left(\frac{\ell}{2}\right)^{2}-\left(z_{n}-c\right)^{2}}
$$

It is easy to check that $z_{n}=w_{+}\left(a_{n}, b_{n}, d_{n}\right)$. If $b>\frac{1}{2} \operatorname{dist}\left(\left[a_{-}, a_{+}\right],\left[d_{-}, d_{+}\right]\right)=\frac{\ell}{2}$, then $b_{n} \leq \frac{\ell}{2}<b$. If $b \leq \frac{\ell}{2}$, then the form of the right-hand side of 3.12 implies that

$$
z_{n} \geq c+\sqrt{\left(\frac{\ell}{2}\right)^{2}-b^{2}}
$$

which yields

$$
b_{n} \leq \sqrt{\left(\frac{\ell}{2}\right)^{2}-\left(\left(\frac{\ell}{2}\right)^{2}-b^{2}\right)}=b .
$$

The relations in 3.13 imply that the operators $A, B$ and $D$ are bounded with $\|B\| \leq b$. If we had $\|B\|<b$, then we would obtain a strictly smaller enclosure for the spectrum from Proposition 3.1. which contradicts the already obtained equality in 3.11) or 3.12, respectively.

\section{Gershgorin-type enclosure for the spectrum of block operator matrices}

In this section we provide another spectral enclosure for the non-real spectrum of the block operator matrix

$$
S=\left[\begin{array}{cc}
A & B \\
-B^{*} & D
\end{array}\right], \quad \operatorname{dom} S=\operatorname{dom} A \oplus \operatorname{dom} D
$$

As already indicated by (4.1), we also allow unbounded entries $A$ and $D$. The operator $B$ remains bounded in our considerations. The result has similarities with Gershgorin's circle theorem for matrices [11] and block operator matrices [27, 28, 29, 9] since we show that the non-real spectrum of the operator matrix $S$ is contained in the union of a family of closed balls, centred along parts of the spectrum of the block $A$ in the diagonal of $S$ (see 4.3 ). To formulate the result, for a closed set $M \subseteq \mathbb{R}$ define the following class of continuous functions:

$$
C^{+}(M)=\left\{f \in C(M): f(t)>0 \text { for } t \in M, \sup _{t \in M} f(t)<\infty, \inf _{|t| \geq 1}|t| f(t)>0\right\} .
$$

The last two conditions obviously only matter if $M$ is unbounded. If $M$ is compact, then $C^{+}(M)$ is the set of positive continuous functions on $M$. Note that any positive constant function is contained in $C^{+}(M)$ and also $|t|^{-1} \in C^{+}(M)$ if $0 \notin M$.

Theorem 4.1. Given a Hilbert space $\mathscr{H}=\mathscr{H}_{+} \oplus \mathscr{H}_{-}$, consider the block operator matrix $S$ in (4.1), where $B \in L\left(\mathscr{H}_{-}, \mathscr{H}_{+}\right)$and $A$ and $D$ are self-adjoint operators in $\mathscr{H}_{+}$and $\mathscr{H}_{-}$, respectively. Then, for 
any $f \in C^{+}(\sigma(A))$ and $g \in C^{+}(\sigma(D))$ we have

$$
\sigma(S) \backslash \mathbb{R} \subseteq\left(\bigcup_{t \in \sigma(A)} \mathscr{B}_{f(t)^{-1}\|f(A) B\|}(t)\right) \cap\left(\bigcup_{s \in \sigma(D)} \mathscr{B}_{g(s)^{-1}\left\|g(D) B^{*}\right\|}(s)\right) .
$$

Remark 4.2. If we set $f=g=1$ in Theorem 4.1, then the spectral inclusion (4.3) becomes

$$
\sigma(S) \backslash \mathbb{R} \subseteq \mathscr{B}_{\|B\|}(\sigma(A)) \cap \mathscr{B}_{\|B\|}(\sigma(D)),
$$

which was already proved in [5, Theorem 3.5].

Theorem 4.1 will follow from Theorem 4.3 below, which is an improvement of [5, Theorem 3.5]. The spectral inclusion (4.4) means that a non-real point in the spectrum of $S$ satisfies $\operatorname{dist}(\lambda, \sigma(A)) \leq\|B\|$ and $\operatorname{dist}(\lambda, \sigma(D)) \leq\|B\|$. This is equivalent to $\left\|(A-\lambda)^{-1}\right\|\|B\| \geq 1$ and $\left\|(D-\lambda)^{-1}\right\|\left\|B^{*}\right\| \geq 1$. Hence, the spectral enclosure given in 4.5 is sharper.

Theorem 4.3. Given a Hilbert space $\mathscr{H}=\mathscr{H}_{+} \oplus \mathscr{H}_{-}$, consider the block operator matrix $S$ in (4.1), where $B \in L\left(\mathscr{H}_{-}, \mathscr{H}_{+}\right)$and $A$ and $D$ are self-adjoint operators in $\mathscr{H}_{+}$and $\mathscr{H}_{-}$, respectively. Then

$$
\sigma(S) \backslash \mathbb{R} \subseteq\left\{\lambda \in \mathbb{C} \backslash \mathbb{R}:\left\|(A-\lambda)^{-1} B\right\| \geq 1 \text { and }\left\|(D-\lambda)^{-1} B^{*}\right\| \geq 1\right\} .
$$

Moreover, given $\lambda \in \mathbb{C} \backslash \mathbb{R}$ then

$$
\begin{array}{ll}
\left\|(S-\lambda)^{-1}\right\| \leq \frac{1+\left\|(A-\lambda)^{-1} B\right\|+\left\|(A-\lambda)^{-1} B\right\|^{2}}{|\operatorname{Im} \lambda| \cdot\left(1-\left\|(A-\lambda)^{-1} B\right\|^{2}\right)} & \text { if }\left\|(A-\lambda)^{-1} B\right\|<1, \\
\left\|(S-\lambda)^{-1}\right\| \leq \frac{1+\left\|(D-\lambda)^{-1} B^{*}\right\|+\left\|(D-\lambda)^{-1} B^{*}\right\|^{2}}{|\operatorname{Im} \lambda| \cdot\left(1-\left\|(D-\lambda)^{-1} B^{*}\right\|^{2}\right)} & \text { if }\left\|(D-\lambda)^{-1} B^{*}\right\|<1 .
\end{array}
$$

Before we prove Theorem 4.3 , we provide a couple of remarks and an example.

Remark 4.4. (a) The same conclusions as in Theorem 4.3 hold, if we drop the boundedness assumption on $B$ and, instead, assume that $B$ is $D$-bounded with $D$-bound less than one and $B^{*}$ is $A$-bounded with $A$-bound less than one. The arguments in the proof are essentially the same.

(b) Note that both $\sigma(S) \backslash \mathbb{R}$ and the right-hand side of 4.5 are sets which are symmetric with respect to the real axis.

(c) There is another spectral enclosure for the operator matrix $S$ that results from a relatively simple argument (see [9, Theorem 1.1] or [4], Lemma 5.2 (ii)]). Consider the second Schur complement $S_{2}(\lambda)=$ $D-\lambda+B^{*}(A-\lambda)^{-1} B$ for $\lambda \in \mathbb{C} \backslash \mathbb{R}$. Applying $(D-\lambda)^{-1}$ from the right and from the left, respectively, we obtain

$$
\begin{aligned}
& S_{2}(\lambda)(D-\lambda)^{-1}=I+B^{*}(A-\lambda)^{-1} B(D-\lambda)^{-1} \quad \text { and } \\
& (D-\lambda)^{-1} S_{2}(\lambda)=I+\left.(D-\lambda)^{-1} B^{*}(A-\lambda)^{-1} B\right|_{\operatorname{dom} D}
\end{aligned}
$$

That is, if one of

$$
N_{S}(\lambda):=\left\|B^{*}(A-\lambda)^{-1} B(D-\lambda)^{-1}\right\| \quad \text { or } \quad N_{S}(\bar{\lambda})=\left\|(D-\lambda)^{-1} B^{*}(A-\lambda)^{-1} B\right\|
$$


is less than 1 , then the Schur complement $S_{2}(\lambda)$ is boundedly invertible and so $\lambda \in \rho(S)$. Hence,

$$
\sigma(S) \subseteq\left\{\lambda \in \rho(D): N_{S}(\lambda) \geq 1 \text { and } N_{S}(\bar{\lambda}) \geq 1\right\} .
$$

A similar reasoning applies to the first Schur complement $S_{1}(\lambda)=A-\lambda+B(D-\lambda)^{-1} B^{*}$ and gives

$$
\sigma(S) \subseteq\left\{\lambda \in \rho(A): M_{S}(\lambda) \geq 1 \text { and } M_{S}(\bar{\lambda}) \geq 1\right\},
$$

where $M_{S}(\lambda):=\left\|B(D-\lambda)^{-1} B^{*}(A-\lambda)^{-1}\right\|$. This implies that

$$
\sigma(S) \backslash \mathbb{R} \subseteq\left\{\lambda \in \mathbb{C} \backslash \mathbb{R}: \min \left\{N_{S}(\lambda), N_{S}(\bar{\lambda}), M_{S}(\lambda), M_{S}(\bar{\lambda})\right\} \geq 1\right\} .
$$

(d) The spectral enclosures (4.5) and (4.8) are independent of each other, meaning that, in general, none of the corresponding sets on the right-hand sides of the two relations contains the other. Consequently, if we intersect the right-hand side of the already known enclosure (4.8) with the new one (4.5), we obtain a better bound for the non-real spectrum of $S$, as illustrated in Example 4.5 below. However, Example 4.9 shows a situation, where (4.5) is in fact strictly better than (4.8).

(e) Both spectral enclosures (4.5) and (4.8) require complete knowledge about the functions $\|(A-$ $\cdot)^{-1} B\|,\|(D-\cdot)^{-1} B^{*} \|, N_{S}$ and $M_{S}$. In contrast, Theorem 4.1 basically only requires knowledge about $\sigma(A)$ and $\sigma(D)$ and is therefore better suited for computations.

Example 4.5. We let $\mathscr{H}_{-}=\mathscr{H}_{+}=\mathbb{C}^{2}$ and

$$
A=\left[\begin{array}{cc}
2 & 1+i \\
1-i & -1
\end{array}\right], \quad D=\left[\begin{array}{cc}
1 & 0 \\
0 & -5
\end{array}\right], \quad B=\left[\begin{array}{cc}
i & 1+\frac{i}{2} \\
-1-i & -\frac{2}{5}
\end{array}\right] .
$$

The four eigenvalues of $S \in \mathbb{C}^{4 \times 4}$ are depicted as black dots in the figure below. Note that two of them are real. They are (approximately) $-4.73166,2.38898,-0.328657 \pm 1.03244 i$. The region from 4.8 , is bounded by the three red curves, while the two blue curves bound the region on the right-hand side of (4.5). The orange filled region is the intersection of the two enclosures.

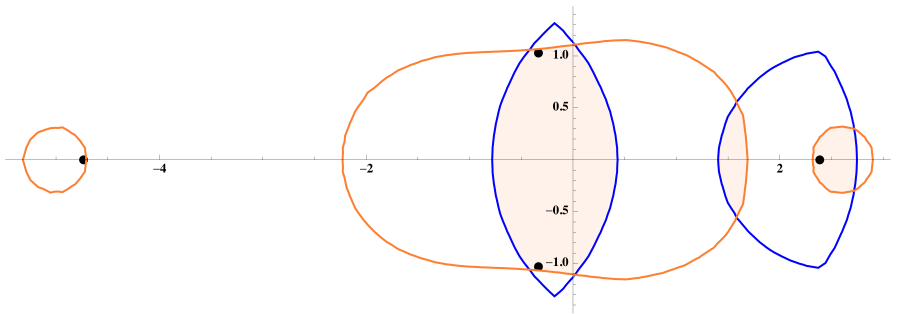

Figure 6: The spectral enclosures 4.5, bounded with blue curves, and 4.8, bounded with orange curves, for the matrix in Example 4.5

Proof of Theorem 4.3. We use the first Schur complement $S_{1}$ of the block operator matrix $S$ in 4.1], which is given by

$$
S_{1}(\lambda)=A-\lambda+B(D-\lambda)^{-1} B^{*}, \quad \operatorname{dom} S_{1}(\lambda)=\operatorname{dom} A,
$$

for $\lambda \in \rho(D)$; see $[2.5$. 
For $\lambda \in \mathbb{C} \backslash \mathbb{R}$ we have $S_{1}(\lambda)^{*}=S_{1}(\bar{\lambda})$ and, setting $T:=(D-\lambda)^{-1} B^{*}$ we obtain

$$
S_{1}(\lambda)-S_{1}(\bar{\lambda})=\bar{\lambda}-\lambda+B\left[(D-\lambda)^{-1}-(D-\bar{\lambda})^{-1}\right] B^{*}=(\lambda-\bar{\lambda})\left(T^{*} T-I\right) .
$$

If $\operatorname{Im} \lambda>0$, then for arbitrary $h \in \operatorname{dom} A$ with $\|h\|=1$ we have

$$
\operatorname{Im}\left(S_{1}(\lambda) h, h\right)=\frac{1}{2 i}\left(\left(S_{1}(\lambda)-S_{1}(\bar{\lambda})\right) h, h\right)=(\operatorname{Im} \lambda) \cdot\left(\|T h\|^{2}-1\right) \leq(\operatorname{Im} \lambda)\left(\|T\|^{2}-1\right) .
$$

In particular, if $\|T\|<1$, then $0 \notin \overline{W\left(S_{1}(\lambda)\right)}$ and therefore $0 \notin \sigma\left(S_{1}(\lambda)\right)$; see Lemma 2.1. Now Lemma 2.2 implies that $\lambda \notin \sigma(S)$. A similar reasoning applies to the case $\operatorname{Im} \lambda<0$. This proves that

$$
\sigma(S) \backslash \mathbb{R} \subseteq\left\{\lambda \in \mathbb{C} \backslash \mathbb{R}:\left\|(D-\lambda)^{-1} B^{*}\right\| \geq 1\right\}
$$

Applying the same arguments to the second Schur complement $S_{2}$, we obtain

$$
\sigma(S) \backslash \mathbb{R} \subseteq\left\{\lambda \in \mathbb{C} \backslash \mathbb{R}:\left\|(A-\lambda)^{-1} B\right\| \geq 1\right\},
$$

which completes the proof of the inclusion 4.5).

Note also that 4.9 implies that

$$
\operatorname{dist}\left(0, \overline{W\left(S_{1}(\lambda)\right)}\right) \geq|\operatorname{Im} \lambda|\left(1-\|T\|^{2}\right)
$$

if $\|T\|<1$. Let us now prove the estimate 4.7) for the resolvent of $S$. For this, let $\lambda \in \mathbb{C} \backslash \mathbb{R}$ such that $\|T\|<1$, where $T=(D-\lambda)^{-1} B^{*}$ as above. By Lemma 2.2 we have

$$
(S-\lambda)^{-1}=\left[\begin{array}{cc}
I & 0 \\
(D-\lambda)^{-1} B^{*} & I
\end{array}\right]\left[\begin{array}{cc}
S_{1}(\lambda)^{-1} & 0 \\
0 & (D-\lambda)^{-1}
\end{array}\right]\left[\begin{array}{cc}
I & -B(D-\lambda)^{-1} \\
0 & I
\end{array}\right]
$$

Denote the first factor by $L$. Then

$$
\begin{aligned}
\|L\|^{2} & =\left\|L^{*} L\right\|=\left\|\left[\begin{array}{cc}
I & T^{*} \\
0 & I
\end{array}\right]\left[\begin{array}{cc}
I & 0 \\
T & I
\end{array}\right]\right\|=\left\|\left[\begin{array}{cc}
I+T^{*} T & T^{*} \\
T & I
\end{array}\right]\right\| \\
& \leq\left\|\left[\begin{array}{ll}
I & 0 \\
0 & I
\end{array}\right]\right\|+\left\|\left[\begin{array}{cc}
T^{*} T & 0 \\
0 & 0
\end{array}\right]\right\|+\left\|\left[\begin{array}{cc}
0 & T^{*} \\
T & 0
\end{array}\right]\right\| \\
& =1+\|T\|^{2}+\|T\| .
\end{aligned}
$$

Since $(D-\lambda)^{-1}$ is normal, we have

$$
\left\|B(D-\lambda)^{-1}\right\|=\left\|(D-\bar{\lambda})^{-1} B^{*}\right\|=\left\|(D-\lambda)^{-1} B^{*}\right\|=\|T\|,
$$

which implies that for the last factor in 4.11 we have the same estimate as for the first one. It remains to estimate the middle factor $M$ in 4.11. To this end, note that Lemma 2.1 and 4.10) yield

$$
\left\|S_{1}(\lambda)^{-1}\right\| \leq \operatorname{dist}\left(0, \overline{W\left(S_{1}(\lambda)\right)}\right)^{-1} \leq|\operatorname{Im} \lambda|^{-1}\left(1-\|T\|^{2}\right)^{-1}
$$

Since $\left\|(D-\lambda)^{-1}\right\| \leq|\operatorname{Im} \lambda|^{-1}$, we obtain

$$
\|M\|=\max \left\{\left\|S_{1}(\lambda)^{-1}\right\|,\left\|(D-\lambda)^{-1}\right\|\right\} \leq|\operatorname{Im} \lambda|^{-1}\left(1-\|T\|^{2}\right)^{-1} .
$$


Hence

$$
\left\|(S-\lambda)^{-1}\right\| \leq \frac{1+\|T\|+\|T\|^{2}}{|\operatorname{Im} \lambda|\left(1-\|T\|^{2}\right)} .
$$

The estimate (4.6) can be derived similarly by using the second Schur complement.

In the following we are going to show that Theorem 4.1 is just a consequence of Theorem 4.3 . However, since the enclosure in Theorem 4.1 is expressed in terms of the spectral quantities of $A$ and $D$, compared with Theorem 4.3 it gives a more intuitive and explicit insight into the location of the spectrum of $S$.

Lemma 4.6. Let $c>0$. Then there exists $C>0$ (depending on $c)$ such that

$$
\left|x^{1+1 / n}-x\right| \leq \frac{C}{n}
$$

for all $x \in[0, c]$ and all $n \in \mathbb{N}$.

Proof. Let $x \in(0, c]$. By the mean value theorem applied to the function $t \mapsto x^{t}$ there exists a $\xi \in\left(1,1+\frac{1}{n}\right)$ such that

$$
x^{1+1 / n}-x=\frac{1}{n} x^{\xi} \log x .
$$

If $x \leq 1$, then

$$
\left|x^{1+1 / n}-x\right| \leq \frac{1}{n} x|\log x| \leq \frac{1}{e n}
$$

If $c>1$ and $x \in(1, c]$, then

$$
\left|x^{1+1 / n}-x\right| \leq \frac{1}{n} x^{1+1 / n} \log x \leq \frac{1}{n} x^{2} \log x \leq \frac{c^{2} \log c}{n} .
$$

This proves the lemma.

Let $\mathscr{H}_{1}$ and $\mathscr{H}_{2}$ be Hilbert spaces, $T$ a self-adjoint operator in $\mathscr{H}_{1}$ and $V \in L\left(\mathscr{H}_{2}, \mathscr{H}_{1}\right)$. Then by $\sigma_{V}(T)$ we denote the support of the positive operator-valued measure $V^{*} E_{T}(\cdot) V$, where $E_{T}$ stands for the spectral measure of $T$. Clearly, $\sigma_{V}(T)$ is a closed subset of $\sigma(T)$. It is compact if and only if $\operatorname{ran} V \subseteq E_{T}(\Delta) \mathscr{H}$ for some bounded set $\Delta \subseteq \mathbb{R}$.

Proposition 4.7. Let $T$ be a self-adjoint operator in $\mathscr{H}_{1}$ and $V \in L\left(\mathscr{H}_{2}, \mathscr{H}_{1}\right)$. Then for $\lambda \in \mathbb{C} \backslash \mathbb{R}$ the following statements are equivalent:

(a) $\left\|(T-\lambda)^{-1} V\right\| \geq 1$;

(b) for all $f \in C^{+}(\sigma(T))$ we have $\lambda \in \bigcup_{t \in \sigma_{V}(T)} \mathscr{B}_{f(t)^{-1}\|f(T) V\|}(t)$.

Proof. (b) $\Rightarrow$ (a). Let $f(t):=|t-\lambda|^{-1}, t \in \sigma(T)$. Then $f \in C^{+}(\sigma(T))$ and thus, by (b), we have $\lambda \in$ $\mathscr{B}_{f\left(t_{0}\right)^{-1}\|f(T) V\|}\left(t_{0}\right)$ for some $t_{0} \in \sigma_{V}(T)$. This means that

$$
\left|t_{0}-\lambda\right| \leq f\left(t_{0}\right)^{-1}\|f(T) V\|=\left|t_{0}-\lambda\right| \cdot\left\|(T-\lambda)^{-1} V\right\|,
$$

which is (a).

(a) $\Rightarrow$ (b). Let $f \in C^{+}(\sigma(T))$. It is obvious that $f$ can be extended to a function in $C^{+}(\mathbb{R})$. Choose such an extension and also denote it by $f$. For $n \in \mathbb{N}$ we set

$$
g_{n}(t):=f(t)|t-\lambda|^{1+1 / n}, \quad t \in \mathbb{R} .
$$


Then each $g_{n}$ is continuous and positive. Note that for $|t| \geq 2|\mu|$, where $\mu=\operatorname{Re} \lambda$, we have $|t-\lambda| \geq$ $|t-\mu| \geq|t|-|\mu| \geq \frac{1}{2}|t|$ and hence $|t-\lambda| f(t) \geq \frac{1}{2}|t| f(t)$, which is bounded below by a positive constant. This implies that $\lim _{|t| \rightarrow \infty}\left|g_{n}(t)\right|=\infty$. Thus, for each $n \in \mathbb{N}$ there exists $t_{n} \in \sigma_{V}(T)$ such that $\left|g_{n}\left(t_{n}\right)\right|=$ $\delta\left(g_{n}\right):=\inf _{t \in \sigma_{V}(T)}\left|g_{n}(t)\right|$.

On the other hand, $\operatorname{dom}|T-\lambda|^{1+1 / n} \subseteq \operatorname{dom} g_{n}(T)$ and $f(T)=g_{n}(T)|T-\lambda|^{-1-1 / n} \in L\left(\mathscr{H}_{1}\right)$. For arbitrary $h \in \mathscr{H}_{2}$ with $\|h\|=1$ define the positive measure $\mu_{h}:=\left\|E_{T}(\cdot) V h\right\|^{2}$, which has support contained in $\sigma_{V}(T)$. Then,

$$
\begin{aligned}
\|f(T) V h\|^{2} & =\left\|g_{n}(T)|T-\lambda|^{-1-1 / n} V h\right\|^{2}=\int_{\sigma_{V}(T)} \frac{\left|g_{n}(t)\right|^{2}}{|t-\lambda|^{2+2 / n}} \mathrm{~d} \mu_{h}(t) \\
& \geq \delta^{2}\left(g_{n}\right)\left\||T-\lambda|^{-1-1 / n} V h\right\|^{2}
\end{aligned}
$$

and hence

$$
\begin{aligned}
\|f(T) V\| & \geq \delta\left(g_{n}\right)\left\||T-\lambda|^{-1-1 / n} V\right\|=\left|g_{n}\left(t_{n}\right)\right|\left\||T-\lambda|^{-1-1 / n} V\right\| \\
& =\left|t_{n}-\lambda\right| f\left(t_{n}\right) \cdot\left|t_{n}-\lambda\right|^{1 / n}\left\||T-\lambda|^{-1-1 / n} V\right\| .
\end{aligned}
$$

Now, consider the functions $h_{n}(t):=|t-\lambda|^{-1-1 / n}, n \in \mathbb{N}$, and $h(t):=|t-\lambda|^{-1}$. Since $|t-\lambda|^{-1} \leq$ $|\operatorname{Im} \lambda|^{-1}$ for all $t \in \mathbb{R}$, it follows from Lemma 4.6 that there exists $C>0$ such that $\left|h_{n}(t)-h(t)\right| \leq C / n$ for all $t \in \mathbb{R}$. This, together with (a), implies that

$$
\begin{aligned}
1-\left\||T-\lambda|^{-1-1 / n} V\right\| & \leq\left\|(T-\lambda)^{-1} V\right\|-\left\||T-\lambda|^{-1-1 / n} V\right\| \\
& =\|h(T) V\|-\left\|h_{n}(T) V\right\| \leq\left\|h(T) V-h_{n}(T) V\right\| \leq \frac{C\|V\|}{n}
\end{aligned}
$$

which, for sufficiently large $n$, yields

$$
\left\||T-\lambda|^{-1-1 / n} V\right\|^{n} \geq\left(1-\frac{C\|V\|}{n}\right)^{n} .
$$

As the right-hand side tends to $e^{-C\|V\|}$ as $n \rightarrow \infty$, there exists $\gamma>0$ such that $\left\||T-\lambda|^{-1-1 / n} V\right\| \geq \gamma^{1 / n}$ for all $n \in \mathbb{N}$. Hence, if there exists some $n \in \mathbb{N}$ such that $\left|t_{n}-\lambda\right| \geq 1 / \gamma$, we find from (4.12) that $\|f(T) V\| \geq\left|t_{n}-\lambda\right| f\left(t_{n}\right)$, which means that $\lambda \in \mathscr{B}_{f\left(t_{n}\right)^{-1}\|f(T) V\|}\left(t_{n}\right)$. Otherwise, there exists a subsequence $\left(t_{n_{k}}\right)$ such that $t_{n_{k}} \rightarrow t_{0}$ as $k \rightarrow \infty$ with $t_{0} \in \sigma_{V}(T)$. In this case, replacing $n$ by $n_{k}$ in (4.12) and letting $k \rightarrow \infty$ we obtain

$$
\|f(T) V\| \geq\left|t_{0}-\lambda\right| f\left(t_{0}\right) \cdot\left\|(T-\lambda)^{-1} V\right\| \geq\left|t_{0}-\lambda\right| f\left(t_{0}\right),
$$

that is, $\lambda \in \mathscr{B}_{f\left(t_{0}\right)^{-1}\|f(T) V\|}\left(t_{0}\right)$.

Proposition 4.7 and Theorem 4.3 now immediately imply the following slight improvement of Theorem 4.1

Theorem 4.8. Let $S$ be the block operator matrix in 4.1. Then, for any $f \in C^{+}(\sigma(A))$ and $g \in C^{+}(\sigma(D))$ we have

$$
\sigma(S) \backslash \mathbb{R} \subseteq\left(\bigcup_{t \in \sigma_{B}(A)} \mathscr{B}_{f(t)^{-1}\|f(A) B\|}(t)\right) \cap\left(\bigcup_{s \in \sigma_{B^{*}}(D)} \mathscr{B}_{g(s)^{-1}\left\|g(D) B^{*}\right\|}(s)\right) .
$$

We shall now check the performance of several spectral enclosures for block operator matrices from above and from the literature on a specific example. The result is illustrated in Figure 7 below. 
Example 4.9. Let $\mathscr{H}_{+}=\mathscr{H}_{-}=\mathbb{C}^{2}$, consider the matrices

$$
A=\left[\begin{array}{ll}
1 & 0 \\
0 & 2
\end{array}\right], \quad B=\left[\begin{array}{cc}
\frac{1}{3} & 0 \\
0 & \frac{2}{3}
\end{array}\right], \quad D=\left[\begin{array}{ll}
1 & 0 \\
0 & 1
\end{array}\right],
$$

and let $S$ be as in 4.1. The eigenvalues of $S$ are given by

$$
1 \pm \frac{1}{3} i \quad \text { and } \quad \frac{3}{2} \pm \frac{\sqrt{7}}{6} i
$$

1. The spectral enclosure from [28, Theorem 2.7] states that

$$
\begin{aligned}
\sigma(S) \backslash \mathbb{R} & \subseteq\left\{\lambda \in \rho(A):\left\|(A-\lambda)^{-1}\right\|^{-1} \leq\|B\|\right\} \cup\left\{\lambda \in \rho(D):\left\|(D-\lambda)^{-1}\right\|^{-1} \leq\|B\|\right\} \\
& =\mathscr{B}_{\frac{2}{3}}(1) \cup \mathscr{B}_{\frac{2}{3}}(2) .
\end{aligned}
$$

2. The spectral enclosure from [27, Theorem 6.4] is slightly better than the previous one:

$$
\sigma(S) \backslash \mathbb{R} \subseteq\left\{\lambda \in \rho(A):\left\|(A-\lambda)^{-1} B\right\| \geq 1\right\} \cup\left\{\lambda \in \rho(D):\left\|(D-\lambda)^{-1} B^{*}\right\| \geq 1\right\} .
$$

However, since

$$
\left\|(A-\lambda)^{-1} B\right\|=\frac{1}{3} \max \left\{|1-\lambda|^{-1}, 2|2-\lambda|^{-1}\right\} \quad \text { and } \quad\left\|(D-\lambda)^{-1} B^{*}\right\|=\frac{2}{3|1-\lambda|}
$$

this yields the same enclosure as before: $\sigma(S) \backslash \mathbb{R} \subseteq \mathscr{B}_{\frac{2}{3}}(1) \cup \mathscr{B}_{\frac{2}{3}}(2)$.

3. The enclosure in [5, Theorem 3.5] (see also (4.4) yields the estimate

$$
\sigma(S) \backslash \mathbb{R} \subseteq\left(\mathscr{B}_{\frac{2}{3}}(1) \cup \mathscr{B}_{\frac{2}{3}}(2)\right) \cap \mathscr{B}_{\frac{2}{3}}(1)=\mathscr{B}_{\frac{2}{3}}(1) .
$$

4. The next enclosure that we check is 4.8 . Since all matrices are diagonal, we have $N(\lambda)=N(\bar{\lambda})=$ $M(\lambda)=M(\bar{\lambda})$. Thus 4.8 is

$$
\sigma(S) \backslash \mathbb{R} \subseteq\left\{\lambda \in \mathbb{C} \backslash \mathbb{R}:\left\|B(D-\lambda)^{-1} B^{*}(A-\lambda)^{-1}\right\| \geq 1\right\} .
$$

We have

$$
B(D-\lambda)^{-1} B^{*}(A-\lambda)^{-1}=\frac{1}{9}\left[\begin{array}{cc}
(1-\lambda)^{-2} & 0 \\
0 & 4(1-\lambda)^{-1}(2-\lambda)^{-1}
\end{array}\right]
$$

and hence

$$
\left\|B(D-\lambda)^{-1} B^{*}(A-\lambda)^{-1}\right\|=\frac{1}{9} \max \left\{\frac{1}{|\lambda-1|^{2}}, \frac{4}{|\lambda-1||\lambda-2|}\right\} .
$$

Therefore a non-real complex number $\lambda$ is in the right-hand side of 4.16 if and only if

$$
9|\lambda-1|^{2} \leq 1 \quad \text { or } \quad 9|\lambda-1||\lambda-2| \leq 4 \text {. }
$$

Since the first inequality implies the second, we obtain that 4.16 is equivalent to

$$
\sigma(S) \backslash \mathbb{R} \subseteq\left\{\lambda \in \mathbb{C} \backslash \mathbb{R}:|\lambda-1||\lambda-2| \leq \frac{4}{9}\right\}
$$


5. To compute (4.5) in Theorem 4.3, we use (4.15) to get

$$
\sigma(S) \backslash \mathbb{R} \subseteq\left(\mathscr{B}_{\frac{1}{3}}(1) \cup \mathscr{B}_{\frac{2}{3}}(2)\right) \cap \mathscr{B}_{\frac{2}{3}}(1)=\mathscr{B}_{\frac{1}{3}}(1) \cup\left(\mathscr{B}_{\frac{2}{3}}(1) \cap \mathscr{B}_{\frac{2}{3}}(2)\right) .
$$

6. Let us now discuss our spectral enclosure from Theorem 4.1. Choose $g(t)=f(t)=|t|^{-1}$, which is valid since $A$ and $D$ are invertible. Then

$$
\|f(A) B\|=\left\|A^{-1} B\right\|=\frac{1}{3} \quad \text { and } \quad\left\|g(D) B^{*}\right\|=\left\|D^{-1} B^{*}\right\|=\frac{2}{3} .
$$

Hence, (4.3) yields

$$
\sigma(S) \backslash \mathbb{R} \subseteq\left(\mathscr{B}_{\frac{1}{3}}(1) \cup \mathscr{B}_{\frac{2}{3}}(2)\right) \cap \mathscr{B}_{\frac{2}{3}}(1)=\mathscr{B}_{\frac{1}{3}}(1) \cup\left(\mathscr{B}_{\frac{2}{3}}(1) \cap \mathscr{B}_{\frac{2}{3}}(2)\right),
$$

which is the same as 4.18.

The right-hand side of 4.18) (or 4.19) is obviously contained in the right-hand side of 4.17;; actually, it is significantly smaller (e.g. the interval $\left(\frac{5}{3}, \frac{7}{3}\right)$ is in the right-hand side of 4.17) but not in the righthand side of (4.19). Note that the first four enclosing sets have the eigenvalues $1 \pm \frac{1}{3} i$ in their interior, while all four eigenvalues of $S$ lie on the boundary of the region given in 4.18).

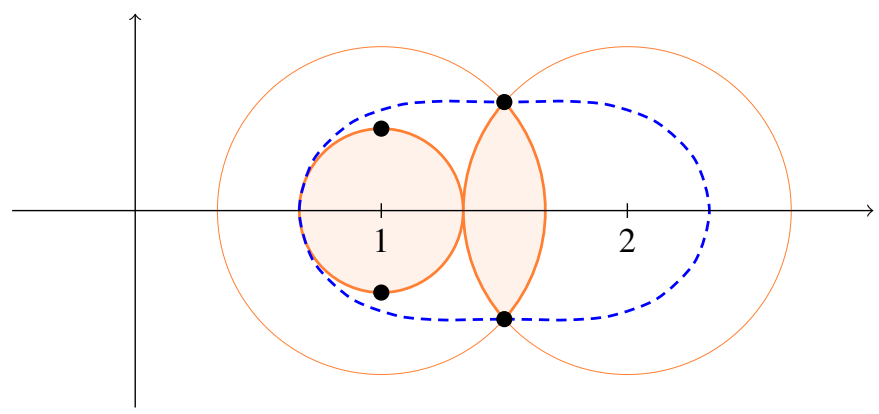

Figure 7: The spectral enclosure for $\sigma(S) \backslash \mathbb{R}$ in 4.18 ) for the operator in Example 4.9 is a union of a disc and the intersection of two discs (filled orange region). The boundary of the set on the right-hand side of 4.17] is the blue dashed line. The eigenvalues of $S$ (see 4.14 ) are indicated with black dots.

In the following corollary we consider a useful special case of Theorem 4.1 We denote by $\mathbb{C}_{+}$and $\mathbb{C}_{-}$ the open right and left half-planes, respectively. The enclosure described in Corollary 4.10 is illustrated in Figure 1 .

Corollary 4.10. Let $S$ be the block operator matrix in 4.1] and assume that $0 \in \rho(A)$. Then

$$
\sigma(S) \backslash \mathbb{R} \subseteq \bigcup_{a \in \sigma_{B}(A)} \mathscr{B}_{|a|\left\|A^{-1} B\right\|}(a) .
$$

Assume, in addition, that $\left\|A^{-1} B\right\|<1$. Then the right-hand side of 4.20 is contained in the set

$$
\left\{z \in \mathbb{C}:|\operatorname{Im} z| \leq \frac{\left\|A^{-1} B\right\|}{\sqrt{1-\left\|A^{-1} B\right\|^{2}}}|\operatorname{Re} z|\right\},
$$


which is a double-sector with half opening angle $\arcsin \left\|A^{-1} B\right\| ;$ moreover,

$$
(\sigma(S) \backslash \mathbb{R}) \cap \mathbb{C}_{+} \subseteq \begin{cases}\left\{z \in \mathbb{C}: \operatorname{Re} z \geq\left(1-\left\|A^{-1} B\right\|\right) \min (\sigma(A) \cap(0, \infty))\right\}, & \sigma(A) \cap(0, \infty) \neq \varnothing \\ \varnothing, & \text { otherwise, }\end{cases}
$$

and

$$
(\sigma(S) \backslash \mathbb{R}) \cap \mathbb{C}_{-} \subseteq \begin{cases}\left\{z \in \mathbb{C}: \operatorname{Re} z \leq\left(1-\left\|A^{-1} B\right\|\right) \max (\sigma(A) \cap(-\infty, 0))\right\}, & \sigma(A) \cap(-\infty, 0) \neq \varnothing \\ \varnothing, & \text { otherwise. }\end{cases}
$$

Proof. Since $0 \in \rho(A)$, the inclusion (4.20) follows from Theorem 4.1 by setting $f(t)=|t|^{-1}$. Now assume also that $\left\|A^{-1} B\right\|<1$. It is elementary to check that the lines

$$
\operatorname{Im} z= \pm \frac{\left\|A^{-1} B\right\|}{\sqrt{1-\left\|A^{-1} B\right\|^{2}}} \operatorname{Re} z
$$

touch the discs $\mathscr{B}_{|a|\left\|A^{-1} B\right\|}(a), a \in \sigma(A)$, tangentially. Further, these discs are contained in the double sector enclosed by the two lines (see (4.21). Hence, the right-hand side of 4.20 ) is contained in 4.21).

Finally, if $\sigma(A) \cap(0, \infty) \neq \varnothing$, then, for every $a \in \sigma(A) \cap(0, \infty)$ and $z \in \mathscr{B}_{a\left\|A^{-1} B\right\|}(a)$, we have

$$
\operatorname{Re} z \geq a-a\left\|A^{-1} B\right\| \geq\left(1-\left\|A^{-1} B\right\|\right) \min (\sigma(A) \cap(0, \infty)) .
$$

Similarly, if $\sigma(A) \cap(-\infty, 0) \neq \varnothing$, then, for every $a \in \sigma(A) \cap(-\infty, 0)$ and $z \in \mathscr{B}_{a\left\|A^{-1} B\right\|}(a)$, we have

$$
\operatorname{Re} z \leq\left(1-\left\|A^{-1} B\right\|\right) \min (\sigma(A) \cap(-\infty, 0)) .
$$

This shows the inclusions for $(\sigma(S) \backslash \mathbb{R}) \cap \mathbb{C}_{+}$and $(\sigma(S) \backslash \mathbb{R}) \cap \mathbb{C}_{-}$.

A similar result holds when one replaces $A$ and $B$ by $D$ and $B^{*}$, respectively. More precisely, if $0 \in \rho(D)$ then

$$
\sigma(S) \backslash \mathbb{R} \subseteq \bigcup_{d \in \sigma_{B^{*}}(D)} \mathscr{B}_{|d||| D^{-1} B^{*} \|}(d) .
$$

If it is also assumed that $\left\|D^{-1} B^{*}\right\|<1$, then

$$
\sigma(S) \backslash \mathbb{R} \subseteq\left\{z \in \mathbb{C}:|\operatorname{Im} z| \leq \frac{\left\|D^{-1} B^{*}\right\|}{\sqrt{1-\left\|D^{-1} B^{*}\right\|^{2}}}|\operatorname{Re} z|\right\}
$$

which is a double-sector with half opening angle $\arcsin \left\|D^{-1} B^{*}\right\|$; moreover,

$$
(\sigma(S) \backslash \mathbb{R}) \cap \mathbb{C}_{+} \subseteq \begin{cases}\left\{z \in \mathbb{C}: \operatorname{Re} z \geq\left(1-\left\|D^{-1} B^{*}\right\|\right) \min (\sigma(D) \cap(0, \infty))\right\}, & \sigma(D) \cap(0, \infty) \neq \varnothing \\ \varnothing, & \text { otherwise, }\end{cases}
$$

and

$$
(\sigma(S) \backslash \mathbb{R}) \cap \mathbb{C}_{-} \subseteq \begin{cases}\left\{z \in \mathbb{C}: \operatorname{Re} z \leq\left(1-\left\|D^{-1} B^{*}\right\|\right) \max (\sigma(D) \cap(-\infty, 0))\right\}, & \sigma(D) \cap(-\infty, 0) \neq \varnothing \\ \varnothing, & \text { otherwise }\end{cases}
$$




\section{Application to $J$-frame operators}

Originally, frame theory has been developed for Hilbert spaces; see, e.g. [7] and the references therein. A frame for a Hilbert space $(\mathscr{H},(\cdot, \cdot))$ is a family of vectors $\mathscr{F}=\left\{f_{i}\right\}_{i \in I}$ for which there exist constants $0<\alpha \leq \beta<\infty$ such that

$$
\alpha\|f\|^{2} \leq \sum_{i \in I}\left|\left\langle f, f_{i}\right\rangle\right|^{2} \leq \beta\|f\|^{2}, \quad \text { for every } f \in \mathscr{H} .
$$

The optimal constants $\alpha$ and $\beta$ for which $(5.1)$ holds are known as the frame bounds of $\mathscr{F}$.

Recently, various approaches have been suggested to introduce frame theory also to Krein spaces; see [10, 13, 26]. In this section we apply our results to $J$-frame operators as introduced in [13]; see also [12]. In particular, we improve the enclosure for the non-real spectrum of $J$-frame operators obtained in [12]; see Theorem 5.2 below.

An indefinite inner product space $(\mathscr{H},[\cdot, \cdot])$ is a (complex) vector space $\mathscr{H}$ endowed with a Hermitian sesquilinear form $[\cdot, \cdot]$. Given a subspace $\mathscr{S}$ of $\mathscr{H}$, the orthogonal subspace to $\mathscr{S}$ is defined by

$$
\mathscr{S}^{[\perp]}=\{x \in \mathscr{H}:[x, s]=0 \text { for every } s \in \mathscr{S}\},
$$

and $\mathscr{S}$ is called non-degenerate if $\mathscr{S} \cap \mathscr{S}^{[\perp]}=\{0\}$. If $\mathscr{S}$ and $\mathscr{T}$ are subspaces of $\mathscr{H}$, the notation $\mathscr{S}[\perp] \mathscr{T}$ stands for $\mathscr{S} \subseteq \mathscr{T}^{[\perp]}$.

A Krein space is a non-degenerate indefinite inner product space $(\mathscr{H},[\cdot, \cdot])$ which admits a decomposition $\mathscr{H}=\mathscr{H}_{+} \dot{+} \mathscr{H}_{-}$such that $\mathscr{H}_{+}[\perp] \mathscr{H}_{-}$and $\left(\mathscr{H}_{ \pm}, \pm[\cdot, \cdot]\right)$ are Hilbert spaces. Such a decomposition is often called a fundamental decomposition and it is denoted $\mathscr{H}=\mathscr{H}_{+}[\dot{+}] \mathscr{H}_{-}$.

The Hilbert spaces $\left(\mathscr{H}_{ \pm}, \pm[\cdot, \cdot]\right)$ induce in a natural way a positive definite inner product $(\cdot, \cdot)$ on $\mathscr{H}$ such that $(\mathscr{H},(\cdot, \cdot))$ is a Hilbert space. Observe that the inner products $[\cdot, \cdot \cdot]$ and $(\cdot, \cdot)$ of $\mathscr{H}$ are related by means of a fundamental symmetry, i.e. a unitary self-adjoint operator $J \in L(\mathscr{H})$ that satisfies

$$
(f, g)=[J f, g], \quad f, g \in \mathscr{H} .
$$

Although the fundamental decomposition is not unique, the norms induced by different fundamental decompositions turn out to be equivalent; see, e.g. [17, Proposition I.1.2]. Therefore, the (Hilbert space) topology in $\mathscr{H}$ does not depend on the chosen fundamental decomposition.

Let us now introduce $J$-frames. Given a Krein space $(\mathscr{H},[\cdot, \cdot])$, consider a frame $\mathscr{F}=\left\{f_{i}\right\}_{i \in I}$ for the associated Hilbert space $(\mathscr{H},(\cdot, \cdot))$ and set

$$
I_{+}:=\left\{i \in I:\left[f_{i}, f_{i}\right] \geq 0\right\} \quad \text { and } \quad I_{-}:=\left\{i \in I:\left[f_{i}, f_{i}\right]<0\right\} .
$$

Then $\mathscr{F}$ is called a $J$-frame for $\mathscr{H}$ if $\mathscr{M}_{+}:=\overline{\operatorname{span}\left\{f_{i}: i \in I_{+}\right\}}$and $\mathscr{M}_{-}:=\overline{\operatorname{span}\left\{f_{i}: i \in I_{-}\right\}}$are nondegenerate subspaces of $\mathscr{H}$ and there exist constants $0<\alpha_{ \pm} \leq \beta_{ \pm}$such that

$$
\alpha_{ \pm}( \pm[f, f]) \leq \sum_{i \in I_{ \pm}}\left|\left[f, f_{i}\right]\right|^{2} \leq \beta_{ \pm}( \pm[f, f]) \quad \text { for } f \in \mathscr{M}_{ \pm}
$$

see [13, Theorem 3.9]. The spaces $\left(\mathscr{M}_{ \pm}, \pm[\cdot, \cdot]\right)$ are then Hilbert spaces by [13, Proposition 3.8] and the optimal constants $0<\alpha_{ \pm} \leq \beta_{ \pm}$are called the $J$-frame bounds of $\mathscr{F}$.

Note that $(5.2)$ says that $\mathscr{F}_{+}=\left\{f_{i}\right\}_{i \in I_{+}}$and $\mathscr{F}_{-}=\left\{f_{i}\right\}_{i \in I_{-}}$are frames for the Hilbert spaces $\left(\mathscr{M}_{+},[\cdot, \cdot]\right)$ and $\left(\mathscr{M}_{-},-[\cdot, \cdot]\right)$, respectively. Moreover, the frame bounds for $\mathscr{F}_{+}$and $\mathscr{F}_{-}$are $\alpha_{+}, \beta_{+}$and $\alpha_{-}, \beta_{-}$, respectively. Also note that not necessarily $\mathscr{M}_{+}[\perp] \mathscr{M}_{-}$. 
The $J$-frame operator associated with $\mathscr{F}$ is defined by

$$
S f=\sum_{i \in I_{+}}\left[f, f_{i}\right] f_{i}-\sum_{i \in I_{-}}\left[f, f_{i}\right] f_{i}, \quad f \in \mathscr{H} .
$$

It plays a fundamental role in the indefinite reconstruction formula (see [13]). The operator $S$ is an invertible, bounded, self-adjoint operator in the Krein space $\mathscr{H}$. The following representation for $J$-frame operators was obtained in [12, Theorems 3.1 and 3.2].

Theorem 5.1. Given a bounded self-adjoint operator $S$ in a Krein space $(\mathscr{H},[\cdot, \cdot])$, the following conditions are equivalent.

(i) $S$ is a J-frame operator.

(ii) There exists a fundamental decomposition

$$
\mathscr{H}=\mathscr{H}_{+}[\dot{+}] \mathscr{H}_{-}
$$

such that $S$ admits a representation with respect to 5.3 of the form

$$
S=\left[\begin{array}{cc}
A & -A K \\
K^{*} A & D
\end{array}\right]
$$

where $A$ is a uniformly positive operator in the Hilbert space $\left(\mathscr{H}_{+},[\cdot, \cdot]\right), K: \mathscr{H}_{-} \rightarrow \mathscr{H}_{+}$is a uniform contraction $\eta^{1}($ i.e. $\|K\|<1)$, and $D$ is a self-adjoint operator such that $D+K^{*} A K$ is uniformly positive in the Hilbert space $\left(\mathscr{H}_{-},-[\cdot, \cdot]\right)$.

(iii) There exists a fundamental decomposition

$$
\mathscr{H}=\mathscr{K}_{+}[\dot{+}] \mathscr{K}_{-}
$$

such that $S$ admits a representation with respect to [5.5) of the form

$$
S=\left[\begin{array}{cc}
A^{\prime} & L D^{\prime} \\
-D^{\prime} L^{*} & D^{\prime}
\end{array}\right]
$$

where $D^{\prime}$ is a uniformly positive operator in $\left(\mathscr{K}_{-},-[\cdot, \cdot]\right), L: \mathscr{K}_{-} \rightarrow \mathscr{K}_{+}$is a uniform contraction 1 , and $A^{\prime}$ is a self-adjoint operator such that $A^{\prime}+L D^{\prime} L^{*}$ is uniformly positive in $\left(\mathscr{K}_{+},[\cdot, \cdot]\right)$.

The representations for the $J$-frame operator given in Theorem 5.1 were used to show that the $J$-frame bounds for $\mathscr{F}$ are related to the boundary of the spectrum of the uniformly positive operators $D+K^{*} A K$ and $A^{\prime}+L D^{\prime} L^{*}$. More precisely, [12, Proposition 4.1] says that if $S$ is represented as in (5.4), then

$$
\alpha_{-}=\min \sigma\left(D+K^{*} A K\right) \quad \text { and } \quad \beta_{-}=\max \sigma\left(D+K^{*} A K\right) .
$$

On the other hand, if $S$ is represented as in 5.6, then

$$
\alpha_{+}=\min \sigma\left(A^{\prime}+L D^{\prime} L^{*}\right) \quad \text { and } \quad \beta_{+}=\max \sigma\left(A^{\prime}+L D^{\prime} L^{*}\right) .
$$

Given a $J$-frame $\mathscr{F}=\left\{f_{i}\right\}_{i \in I}$ for $\mathscr{H}$ with $J$-frame operator $S$, the canonical dual $J$-frame of $\mathscr{F}$ is defined as $\mathscr{F}^{\prime}=\left\{S^{-1} f_{i}\right\}_{i \in I}$. It is also a $J$-frame for $\mathscr{H}$ such that $\mathscr{F}_{ \pm}^{\prime}=\left\{S^{-1} f_{i}\right\}_{i \in I_{ \pm}}$are frames for

${ }^{1}$ The operator norm used depends on the norm induced by the respective fundamental decomposition 5.3 or 5.5 . 
$\left(\mathscr{M}_{\mp}^{[\perp]}, \pm[\cdot, \cdot]\right)$, i.e. there exist constants $0<\gamma_{ \pm} \leq \delta_{ \pm}$such that

$$
\gamma_{ \pm}( \pm[f, f]) \leq \sum_{i \in I_{ \pm}}\left|\left[f, S^{-1} f_{i}\right]\right|^{2} \leq \delta_{ \pm}( \pm[f, f]) \quad \text { for every } f \in \mathscr{M}_{\mp}^{[\perp]} .
$$

The $J$-frame bounds of $\mathscr{F}^{\prime}$ are also related to the representations in Theorem 5.1 if $S$ is represented as in (5.6) then

$$
\gamma_{-}=\min \sigma\left(\left(D^{\prime}\right)^{-1}\right)=\left(\max \sigma\left(D^{\prime}\right)\right)^{-1} \quad \text { and } \quad \delta_{-}=\max \sigma\left(\left(D^{\prime}\right)^{-1}\right)=\left(\min \sigma\left(D^{\prime}\right)\right)^{-1},
$$

and if $S$ is represented as in 5.4 then

$$
\gamma_{+}=\min \sigma\left(A^{-1}\right)=(\max \sigma(A))^{-1} \quad \text { and } \quad \delta_{+}=\max \sigma\left(A^{-1}\right)=(\min \sigma(A))^{-1} ;
$$

see [12, Proposition 4.2].

The following theorem gives an enclosure for the non-real spectrum of the $J$-frame operator $S$ of a $J$-frame $\mathscr{F}$ in terms of the $J$-frame bounds associated with $\mathscr{F}$ and its canonical dual $J$-frame $\mathscr{F}^{\prime}$.

Theorem 5.2. Let $\mathscr{F}$ be a J-frame for $(\mathscr{H},[\cdot, \cdot])$ with J-frame operator $S$. Then,

$$
\sigma(S) \backslash \mathbb{R} \subseteq\left(\bigcup_{a \in\left[\delta_{+}^{-1}, \gamma_{+}^{-1}\right]} \mathscr{B}_{a\|K\|}(a)\right) \cap\left(\bigcup_{b \in\left[\alpha_{-}, \beta_{-}\right]} \mathscr{B}_{\frac{b\|K\|}{1-\|K\|^{2}}}\left(\frac{b}{1-\|K\|^{2}}\right)\right),
$$

where $K$ is the angular operator appearing in (5.4). Also,

$$
\sigma(S) \backslash \mathbb{R} \subseteq\left(\bigcup_{d \in\left[\delta_{-}^{-1}, \gamma_{-}^{-1}\right]} \mathscr{B}_{d\|L\|}(d)\right) \cap\left(\bigcup_{b \in\left[\alpha_{+}, \beta_{+}\right]} \mathscr{B}_{\frac{b\|L\|}{1-\|L\|^{2}}}\left(\frac{b}{1-\|L\|^{2}}\right)\right),
$$

where $L$ is the angular operator appearing in 5.6. The sets on the right-hand sides of (5.11) and (5.12) are contained in sectors of the form $\left\{z \in \mathbb{C}_{+}:|\operatorname{Im} z| \leq \tan \varphi \cdot \operatorname{Re} z\right\}$ with half opening angles $\varphi=\arcsin \|K\|$ and $\varphi=\arcsin \|L\|$, respectively.

Proof. Let $\sigma_{B}(A)$ be defined as before Proposition 4.7. Obviously we have $\sigma_{B}(A) \subseteq \sigma(A) \subseteq\left[a_{-}, a_{+}\right]$, where the constants $a_{ \pm}$are defined in 3.1. Applying Corollary 4.10 to $S$ represented as in (5.4) we obtain that

$$
\sigma(S) \backslash \mathbb{R} \subseteq \bigcup_{a \in\left[a_{-}, a_{+}\right]} \mathscr{B}_{a\|K\|}(a) .
$$

Moreover, according to 5.10) we have that $a_{-}=\delta_{+}^{-1}$ and $a_{+}=\gamma_{+}^{-1}$. On the other hand, $S^{-1}$ is also a $J$-frame operator and it is easy to check that

$$
S^{-1}=\left[\begin{array}{cc}
A^{-1}-K Z K^{*} & K Z \\
-Z K^{*} & Z
\end{array}\right],
$$

where $Z:=\left(D+K^{*} A K\right)^{-1}$ is a uniformly positive operator; cf. Theorem 5.1 Therefore Corollary 4.10 applied to $S^{-1}$ represented as above implies

$$
\sigma\left(S^{-1}\right) \backslash \mathbb{R} \subseteq \bigcup_{r \in\left[r_{-}, r_{+}\right]} \mathscr{B}_{r\left\|K^{*}\right\|}(r)
$$


where $\left[r_{-}, r_{+}\right]$is the closure of the numerical range of $Z$. Also, 5.7 says that $r_{-}=\beta_{-}^{-1}$ and $r_{+}=\alpha_{-}^{-1}$. With $b:=\frac{1}{r}$ it follows that

$$
\sigma\left(S^{-1}\right) \backslash \mathbb{R} \subseteq \bigcup_{b \in\left[\alpha_{-}, \beta_{-}\right]} \mathscr{B}_{\frac{\|K\|}{b}}\left(\frac{1}{b}\right)
$$

Recall that $\lambda \in \sigma(S) \backslash\{0\}$ if and only if $\frac{1}{\lambda} \in \sigma\left(S^{-1}\right) \backslash\{0\}$. Moreover, observe that for $r>0$

$$
\frac{1}{\lambda} \in \mathscr{B}_{\frac{\|K\|}{r}}\left(\frac{1}{r}\right) \quad \text { if and only if } \quad \lambda \in \mathscr{B} \frac{r\|K\|}{1-\|K\|^{2}}\left(\frac{r}{1-\|K\|^{2}}\right) .
$$

Therefore,

$$
\sigma(S) \backslash \mathbb{R} \subseteq \bigcup_{b \in\left[\alpha_{-}, \beta_{-}\right]} \mathscr{B} \frac{b\|K\|}{1-\|K\|^{2}}\left(\frac{b}{1-\|K\|^{2}}\right),
$$

and (5.11) follows by intersecting (5.13) and (5.14).

The proof of (5.12) is similar. It follows from Corollary 4.10 applied to $S$ represented as in (5.6), and also to $S^{-1}$ represented as

$$
S^{-1}=\left[\begin{array}{cc}
Z^{\prime} & -Z^{\prime} L \\
L^{*} Z^{\prime} & \left(D^{\prime}\right)^{-1}-L^{*} Z^{\prime} L
\end{array}\right]
$$

with $Z^{\prime}=\left(A^{\prime}+L D^{\prime} L^{*}\right)^{-1}$.

The statement about the sectors is clear from Corollary 4.10

In the following, we compare Theorem 5.2 with the enclosure for the non-real spectrum of $J$-frame operators obtained in [12].

Let $\mathscr{F}$ be a $J$-frame for a Krein space $(\mathscr{H},[\cdot, \cdot])$ with $J$-frame operator $S$ and $J$-frame bounds $0<$ $\alpha_{ \pm} \leq \beta_{ \pm}$. Assume also that $0<\gamma_{ \pm} \leq \delta_{ \pm}$are the $J$-frame bounds of its canonical dual $J$-frame $\mathscr{F}^{\prime}$. In [12, Corollary 5.3] it was shown that

$$
\sigma(S) \backslash \mathbb{R} \subseteq{\stackrel{\mathscr{B}}{\min }\left\{\gamma_{+}^{-1}, \gamma_{-}^{-1}\right\}}\left(\min \left\{\gamma_{+}^{-1}, \gamma_{-}^{-1}\right\}\right) \cap\left\{\lambda \in \mathbb{C}: \operatorname{Re} \lambda \geq \frac{\max \left\{\alpha_{+}, \alpha_{-}\right\}}{2}\right\}
$$

Here, $\mathscr{\mathscr { B }}_{r}(a)$ denotes the interior of $\mathscr{B}_{r}(a)$. Let us show that the intersection of the sets on the right-hand sides of 5.11) and 5.12) are (strictly) contained in the right-hand side of 5.15.

For every $a \in\left[\delta_{+}^{-1}, \gamma_{+}^{-1}\right]$ it is easy to see that $\mathscr{B}_{a\|K\|}(a)$ is strictly contained in ${\stackrel{\mathscr{B}}{\gamma_{+}^{-1}}}\left(\gamma_{+}^{-1}\right)$. Therefore,

$$
\bigcup_{a \in\left[\delta_{+}^{-1}, \gamma_{+}^{-1}\right]} \mathscr{B}_{a\|K\|}(a) \subseteq \stackrel{\circ}{\mathscr{B}}_{\gamma_{+}^{-1}}\left(\gamma_{+}^{-1}\right)
$$

On the other hand, given $r>0$, if $\lambda \in \mathscr{B} \frac{r\|K\|}{1-\|K\|^{2}}\left(\frac{r}{1-\|K\|^{2}}\right)$, then $\operatorname{Re} \lambda \geq \frac{r}{1+\|K\|}>\frac{r}{2}$. Thus,

$$
\bigcup_{b \in\left[\alpha_{-}, \beta_{-}\right]} \mathscr{B}_{\frac{b\|K\|}{1-\|K\|^{2}}}\left(\frac{b}{1-\|K\|^{2}}\right) \subseteq\left\{\lambda \in \mathbb{C}: \operatorname{Re} \lambda \geq \frac{\alpha_{-}}{2}\right\}
$$

Similarly, it is easy to see that

$$
\bigcup_{d \in\left[\delta_{-}^{-1}, \gamma_{-}^{-1}\right]} \mathscr{B}_{d\|L\|}(d) \subseteq \stackrel{\circ}{\mathscr{B}}_{\gamma_{-}^{-1}}\left(\gamma_{-}^{-1}\right)
$$


and

$$
\left(\bigcup_{b \in\left[\alpha_{+}, \beta_{+}\right]} \mathscr{B} \frac{b\|L\|}{1-\|L\|^{2}}\left(\frac{b}{1-\|L\|^{2}}\right)\right) \subseteq\left\{\lambda \in \mathbb{C}: \operatorname{Re} \lambda \geq \frac{\alpha_{+}}{2}\right\} .
$$

Hence, Theorem 5.2 improves the enclosure 5.15 for the non-real spectrum of the $J$-frame operator $S$ obtained in [12].

\section{Acknowledgements}

F. Martínez Pería and C. Trunk gratefully acknowledge the support of the DFG (Deutsche Forschungsgemeinschaft) from the project TR 903/21-1. In addition, J. I. Giribet and F. Martínez Pería gratefully acknowledges the support from the grant PIP CONICET 0168.

\section{References}

[1] A. Adamjan and H. Langer, Spectral properties of a class of rational operator valued functions, $J$. Operator Theory 33 (1995), 259-277.

[2] S. Albeverio and A. K. Motovilov, On invariant graph subspaces of a $J$-self-adjoint operator in the Feshbach case, Math. Notes 100 (2016), 761-773.

[3] S. Albeverio, A. K. Motovilov and A. A. Shkalikov, Bounds on variation of spectral subspaces under $J$-self-adjoint perturbations, Integral Equations Operator Theory 64 (2009), 455-486.

[4] S. Albeverio, A. K. Motovilov and C. Tretter, Bounds on the spectrum and reducing subspaces of a $J$-self-adjoint operator, Indiana Univ. Math. J. 59 (2010), 1737-1776.

[5] J. Behrndt, F. Philipp and C. Trunk, Bounds on the non-real spectrum of differential operators with indefinite weights, Math. Ann. 357 (2013), 185-213.

[6] E. P. Bogomolova, Some questions of the spectral analysis of a nonselfadjoint differential operator with a "floating" singularity in the coefficient, Differential Equations 21 (1985), 1229-1234.

[7] O. Christensen, An Introduction to Frames and Riesz Bases, Birkhäuser, Boston, 2003.

[8] J.-C. Cuenin and C. Tretter, Non-symmetric perturbations of self-adjoint operators, J. Math. Anal. Appl. 441 (2016), 235-258.

[9] A. Dall'Aqua, D. Mugnolo and M. Schelling, A new Gershgorin-type result for the localisation of the spectrum of matrices, Math. Nach. 288 (2015), 1981-1994.

[10] K. Esmeral, O. Ferrer and E. Wagner, Frames in Krein spaces arising from a non-regular $W$-metric, Banach J. Math. Anal. 9 (2015), 1-16.

[11] S. Gershgorin, Über die Abgrenzung der Eigenwerte einer Matrix, Bull. Acad. des Sci. URSS 6 (1931), 749-754 [German].

[12] J. I. Giribet, M. Langer, L. Leben, A. Maestripieri, F. Martínez Pería and C. Trunk, Spectrum of $J$-frame operators, Opuscula Math. 38 (2018), 623-649.

[13] J. I. Giribet, A. Maestripieri, F. Martínez Pería and P. G. Massey, On frames for Krein spaces, $J$. Math. Anal. Appl. 393 (2012), 122-137. 
[14] P. Jonas and C. Trunk, On a class of analytic operator functions and their linearizations, Math. Nachr. 243 (2002), 92-133.

[15] P. Jonas and C. Trunk, A Sturm-Liouville problem depending rationally on the eigenvalue parameter, Math. Nachr. 280 (2007), 1709-1726.

[16] T. Kato, Perturbation Theory for Linear Operators, Springer-Verlag, Berlin-Heidelberg, 1995.

[17] H. Langer, Spectral functions of definitizable operators in Krein spaces, in: Functional Analysis. (Proceedings of a Conference held at Dubrovnik, 1981.) Lecture Notes in Math., vol. 948, Springer, Berlin-New York, 1982, pp. 1-46.

[18] H. Langer, M. Langer, A. Markus and C. Tretter, Spectrum of definite type of self-adjoint operators in Krein spaces, Linear Multilinear Algebra 53 (2005), 115-136.

[19] H. Langer, M. Langer and C. Tretter, Variational principles for eigenvalues of block operator matrices, Indiana Univ. Math. J. 51 (2002), 1427-1459.

[20] H. Langer, A. Markus and V. Matsaev, Locally definite operators in indefinite inner product spaces, Math. Ann. 308 (1997), 405-424.

[21] H. Langer, R. Mennicken and M. Möller, A second order differential operator depending nonlinearly on the eigenvalue parameter, in: Topics in Operator Theory. Ernst D. Hellinger Memorial Volume. Oper. Theory Adv. Appl., vol. 48, Birkhäuser, Basel, 1990, pp. 319-332.

[22] H. Langer and C. Tretter, Spectral decomposition of some nonselfadjoint block operator matrices, J. Operator Theory 39 (1998), 339-359.

[23] M. Langer and M. Strauss, Spectral properties of unbounded J-self-adjoint block operator matrices, J. Spectr. Theory 7 (2017), 137-190.

[24] R. Mennicken and A. A. Shkalikov, Spectral decomposition of symmetric operator matrices, Math. Nachr. 179 (1996), 259-273.

[25] R. Nagel, Towards a "Matrix Theory" for Unbounded Operator Matrices, Math. Z. 201 (1989), 57-68.

[26] I. Peng and S. Waldron, Signed frames and Hadamard products of Gram matrices, Linear Algebra Appl. 347 (2002), 131-157.

[27] T. H. Rasulov and C. Tretter, Spectral inclusion for unbounded diagonally dominant $n \times n$ operator matrices, Rocky Mountain J. Math. 48 (2018), 279-324.

[28] H. N. Salas, Gershgorin's theorem for matrices of operators, Lineal Algebra Appl. 291 (1999), 1536.

[29] C. Tretter, Spectral Theory of Block Operator Matrices and Applications, Imperial College Press, London, 2008.

[30] C. Tretter, Spectral inclusion for unbounded block operator matrices, J. Funct. Anal. 256 (2009), 3806-3829. 